Wilfried E. Keil

\title{
Schrift und Bild zur Bildung?
}

\author{
Die Kapitelle im Kreuzgang von SS. Pietro ed Orso in Aosta
}

Kreuzgänge sind nicht nur Verbindungswege zwischen an sie angebaute architektonische Räume, sondern auch von sakralen Räumen wie der Kirche zu sozialen Räumen wie den Konventsgebäuden, die in Teilen zugleich auch sakrale Räume sein können. Zudem sind sie selbst architektonischer und sozialer Raum, der ephemer durch Prozessionen zu einem Sakralraum und liturgischen Raum werden kann. Ein Kreuzgang ist also nicht nur ein Verkehrsweg von Klerikern. Üblicherweise sind Kreuzgänge durch baugebundene Skulpturen geschmückt, manche sogar mit figürlicher Bauskulptur. Einige Kreuzgänge weisen auch Inschriften auf, die nicht auf eingebauten Grabplatten und Grabmälern stehen, sondern an Portalen, Reliefplatten, Pfeilern oder Kapitellen. Hierbei kommt es in vielen Fällen zu einer Verbindung von Schrift und Bild. Die Inschriften sind hierbei nicht isoliert zu betrachten, und auch nicht nur gemeinsam mit den Bildwerken, sondern auch die Anordnung im Raum ist zu berücksichtigen, da diese entscheidend für die Funktion, Wahrnehmung und Bedeutung der Inschriften und Bildwerke ist. ${ }^{1}$

Wieso wurden in manchen Kreuzgängen zusätzlich zur Skulptur Inschriften angebracht, die über Beischriften hinausgehen und hiermit mehr als das Dargestellte zum Thema haben? Folgt die Anordnung der Inschriften einem bestimmten Konzept, das in die Verkehrswege und Raumfunktionen eingebunden ist?

Für erste Überlegungen zu Kreuzgängen als Schriftraum dient im Folgenden der romanische Kreuzgang der Stiftskirche SS. Pietro ed Orso in Aosta mit seinen Kapitellen, die teilweise mit Inschriften unterschiedlicher Art verstehen sind (Abb.1). ${ }^{2}$

\footnotetext{
1 Für die Wichtigkeit der Anordnung von Inschriften siehe: Debiais 2009, 65-91. Auch innerhalb der Räume ist die topologische Anordnung der Inschriften zu berücksichtigen. Siehe hierzu: Dickmann/ Keil/Witschel 2015. Auch eine abgestufte Sakralhierarchie innerhalb eines Sakralraums ist möglich. Zu Überlegungen hierzu anhand von Fußbodenmosaiken im Frühchristentum siehe: Jäggi 2007, 80-89. 2 Die Aussage von Joachim Poeschke, dass der Kreuzgang von Aosta neben dem von Monreale der einzige romanische mit figürlichem Kapitellschmuck in Italien sei, trifft nicht zu. Siehe: Poeschke 1998, 97. Zum Kreuzgang von SS. Pietro ed Orso in Aosta siehe vor allem: Berton 1956; Barberi 1988; Barberi 2001. Die Kapitelle des Kreuzgangs wurden im Rahmen des CENOBIUM-Projekts in 3D gescannt. Siehe hierzu: Dercks 2012.
}

Dieser Beitrag ist im Heidelberger Sonderforschungsbereich 933 „Materiale Textkulturen. Materialität und Präsenz des Geschriebenen in non-typographischen Gesellschaften“ entstanden (Teilprojekt A05 „Schrift und Schriftzeichen am und im mittelalterlichen Kunstwerk“). Der SFB 933 wird durch die Deutsche Forschungsgemeinschaft finanziert. Alle Transkriptionen und Übersetzungen stammen vom Verfasser.

Ә Open Access. () 2019 Wilfried E. Keil, publiziert von De Gruyter. (c) BY-NC-ND Dieses Werk ist lizenziert unter der Creative Commons Attribution-NonCommercial-NoDerivatives 4.0 Lizenz. 


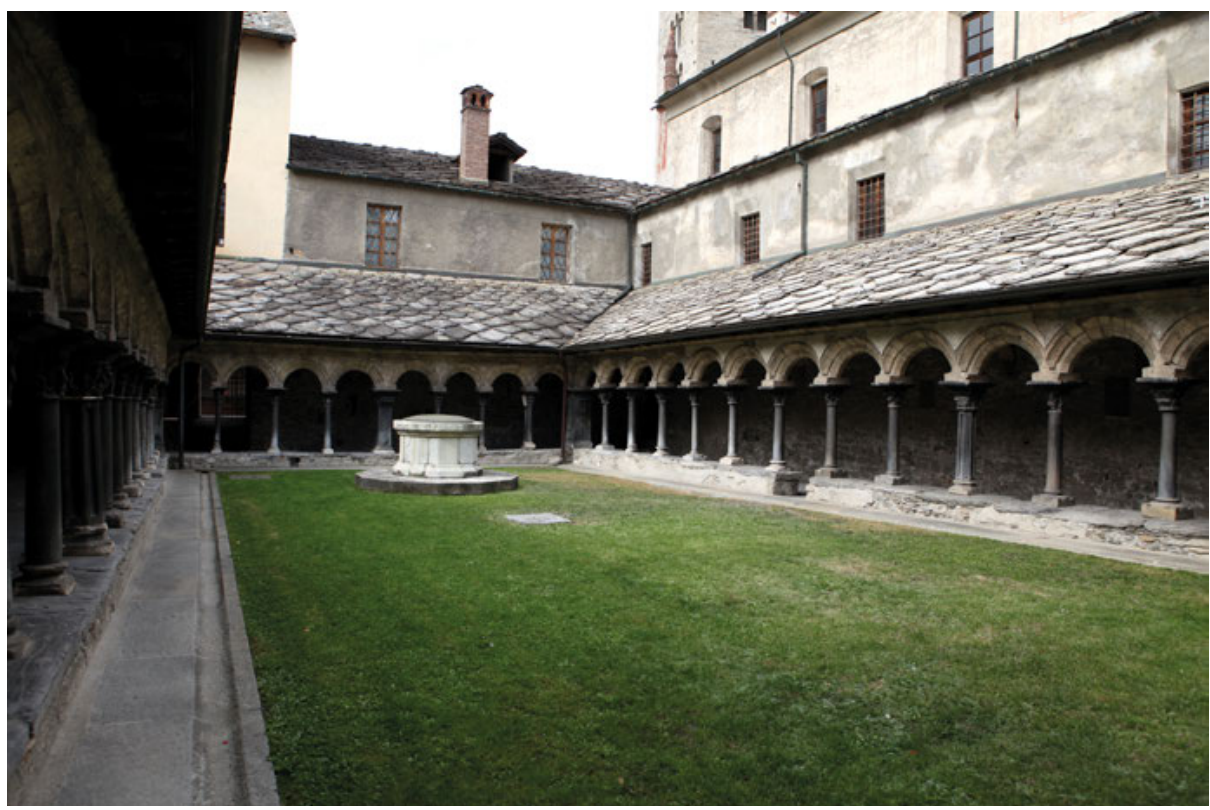

Abb.1: Aosta, Santi Pietro ed Orso, Kreuzgang, Sicht nach Westen.

Aosta im Aostatal in Oberitalien liegt an einer alten Römerstraße. Die heutige Stadtanlage beruht immer noch auf den römischen Strukturen. Aosta war seit dem Frühmittelalter eine bedeutende Station auf dem Pilgerweg nach Rom, der Via Francigena, die von Canterbury über Reims kommend, über Pavia und Lucca nach Rom weiterführte.

Unter der Kirche von Santi Pietro ed Orso wurden zahlreiche Bestattungen in den Resten frühchristlicher Vorgängerbauten gefunden. Hierbei kam ein Inschriftenfragment zu Tage, das zu einer Bestattung eines Bischofs im Jahre 522 gehörte. ${ }^{3}$ Auch von einem karolingischen Bau sind Mauerreste und Relieffragmente erhalten. Vom 10. bis 12. Jahrhundert wurde die Kirche mehrfach erweitert und umgestaltet. ${ }^{4}$

1133, nach heutiger Zeitrechnung 1132, wurde das Kapitel reformiert und durch Papst Innozenz II. als Augustinerchorherrenkonvent bestätigt. ${ }^{5}$ Treibende Kraft hierbei war der damalige Bischof von Aosta, Aribert (1132-1139), der selbst ein Augustinerchorherr war und aus der Abtei von Sainte-Marie-d'Abondance im Chablais (Département Haute-Savoie) kam. Der erste Prior, Arnulf von Avise, wurde später selbst Bischof von Aosta (1149-1159). ${ }^{6}$

3 Bonnet/Perinetti 2001, $15 \mathrm{f}$.

$4 \mathrm{Zu}$ einem Überblick von den antiken Funden bis zu den Umbauten im 13. Jahrhundert siehe: Bonnet/Perinetti 2001.

5 Bonnet/Perinetti 2001, 23.

6 Barberi 2001, 49. 
Der romanische Kreuzgang liegt südlich der Kirche. Die Datierung des Kreuzgangs und seiner Kapitelle ist umstritten. Ein Teil der Forscher ist der Meinung, dass die Kapitelle direkt nach der Übertragung an die Augustinerchorherren 1132 entstanden sind $;^{7}$ andere datieren sie nach der Mitte des 12. Jahrhunderts, als der frühere erste Prior Arnulf Bischof von Aosta war. Für die Spätdatierung gibt es zwei Argumente. Das eine beruht auf stilistischen Vergleichen und das andere auf dem Kapitell, das die Übergabe der Augustinusregel zum Thema hat. Francesco Gandolfo will die Inschrift EPISCOPVS, die sich auf der Kapitellseite mit Augustinus befindet, dem Prior Arnulf, der fast auf der Ecke, am Übergang von der einen zur anderen Seite steht, der zum Prior gehörigen Inschrift auf der anderen Kapitellseite zuordnen, um seine Spätdatierung zu rechtfertigen. ${ }^{8}$ Diese Argumentation ist nicht nachzuvollziehen, da die Bezeichnung klar auf den im Bischofsornat dargestellten Augustinus, der Bischof von Hippo Regius war, zu beziehen ist. ${ }^{9}$ Wenn die Kapitelle zur Zeit Arnulfs als Bischof entstanden wären, wäre dies in der sonst im gesamten Kreuzgang angewandten Weise mitgeteilt worden: Die Beischriften stehen immer auf der Seite, auf der das Benannte dargestellt ist; also wird es in diesem Fall auch so sein. Zudem wäre dem Konzepteur damals klar gewesen, dass man daher das EPISCOPVS auf Augustinus bezogen hätte.

Charles Bonnet und Renato Perinetti ordnen unabhängig von Francesco Gandolfo die Kapitelle in das fünfte Jahrzehnt des 12. Jahrhunderts ein. ${ }^{10}$ Joachim Poeschke datiert die Kapitelle auf Grund stilistischer Ähnlichkeiten mit Saint-Trophime in Arles und der Kathedrale von Avignon um 1150/60. ${ }^{11}$ Nach Poeschke gibt es bis auf einige Fragmente im Dom von Aosta im ganzen Aostatal und im benachbarten Piemont keine Vergleichsbeispiele. ${ }^{12}$ Insgesamt können die Versuche der Forschung, die Kapitelle von Aosta stilistisch einzuordnen, alle nicht wirklich überzeugen. Einzig René Jullians Zuweisung der im 19. Jahrhundert im Baptisterium von Saint-Martin d'Ainay in Lyon wiederverwendeten Kapitelle an die Werkstatt des Kreuzgangs von SS. Pietro ed Orso in Aosta erscheint einleuchtend. ${ }^{13}$

Im Laufe der Jahrhunderte kam es immer wieder zu Restaurierungen und Veränderungen am Kreuzgang. Die hölzernen Dächer der Kreuzgangflügel wurden Quellennachrichten zufolge unter Prior Jean de Champvillar im Jahre 1364 durch steinerne

7 Barberi 2001, 57f. Zu weiteren stilistischen Einordnungen mit weiterer Literatur siehe: Barberi 2001, 58-64. Zur Datierungsproblematik und weiterer Literatur hierzu siehe: Barberi 1988, 29f. Zum Stil der Kapitelle und weiteren stilistischen Vergleichen siehe: Barberi 1988, 35-83.

8 Gandolfo 1999. Lucy Donkin folgt der Argumentation von Francesco Gandolfo. Siehe: Donkin 2008, 78.

9 Diesen logischen Bezug hatte bereits Arthur Kingsley Porter gemacht. Siehe: Porter 1915-1917, Bd.2, 58.

10 Bonnet/Perinetti 2001, 23, 30.

11 Poeschke 1998, 98.

12 Poeschke 1998, 97. Zu den Beispielen siehe: Barberi 1988, 41-46.

13 Jullian 1964. 
Gewölbe ersetzt. ${ }^{14} 1419$ ordnete Bischof Oger Moriset eine Restaurierung des verfallenen und ruinösen Kreuzgangs an. Bereits 1427 mussten die Dächer neu gedeckt werden. ${ }^{15} 1644$ wurde das Kollegiatsstift säkularisiert. ${ }^{16}$ Ende des 17 . Jahrhunderts mussten die Dächer des Kreuzgangs wiederum erneuert werden. ${ }^{17} 1778$ wurden die beiden Architekten Albertolli und Franchino damit beauftragt, ein größeres Gebäude an der Stelle zu errichten, an der früher das Dormitorium der Kanoniker im Ostflügel lag. ${ }^{18}$ Dieses Gebäude wurde im Jahre 1826 wieder abgerissen und ein neues Gebäude für die Kanoniker errichtet. Dabei wurden die Gewölbe des Kreuzgangostflügels erneuert. ${ }^{19}$ Nur das mittlere Kapitell des Ostflügels mit vegetabilen Formen ist noch ursprünglich. ${ }^{20}$ Von den nicht wiederverwendeten Kapitellen gelangten einige im Laufe der Zeit über Umwege ins Museo Civico di Torino. ${ }^{21}$

Weitere Restaurierungen fanden im 19. und 20. Jahrhundert statt. Ein Hinweis hierauf ist z. B. eine Bodenplatte in der Nordostecke des Kreuzgangs, in die die Jahreszahl 1905 eingehauen ist. Die Platte wurde später teilweise überbaut.

Von den ursprünglich 52 Kapitellen im Kreuzgang blieben 42 erhalten. Vier davon kamen Ende des 19. Jahrhunderts in das Museo Civico di Torino. ${ }^{22}$ Durch die vielen Restaurierungen und Umbauten sind weder die Frage, inwieweit der heutige Zustand dem ursprünglichen entspricht, noch die ursprüngliche Anordnung der Kapitelle wie bei vielen anderen Kreuzgängen ohne eine genaue Untersuchung zu klären. ${ }^{23}$ Allerdings finden sich auf einigen Basen, Säulen, Kapitellen und Kämpferplatten Versatzmarken. Ob diese dem ursprünglichen Steinversatz dienten oder von Restaurierungsarbeiten stammen, konnte bisher noch nicht geklärt werden.

Allerdings wurden die Kapitelle bei Umbauten wohl nicht weit entfernt deponiert, und es ist recht wahrscheinlich, dass die eines Kreuzgangflügels gemeinsam gelagert wurden. Zumindest bilden die Kapitelle der einzelnen Flügel eine Einheit. Daher ist anzunehmen, dass nur Kapitelle innerhalb eines Kreuzgangflügels vertauscht oder gedreht und in veränderter Ausrichtung wieder eingebaut wurden.

Die Kapitelle sowie ein Teil der Basen wurden aus weißem Marmor gefertigt und die Schäfte sowie die meisten Basen aus Bardiglio-Marmor aus Aymavilles, der graugrün marmoriert ist. ${ }^{24}$ Die Kapitelle wurden nachträglich schwarz gefasst. Dies war

\footnotetext{
14 Barberi 2001, 50.

15 Barberi 1988, 22; Barneri 2001, 51.

16 Barberi 1988, 22; Barberi 2001, 51.

17 Barberi 1988, 22; Barberi 2001, 51.

18 Barberi 1988, 23 u. Anm. 16; Barberi 2001, 51.

19 Barberi 2001, 51.

20 Barberi 2001, 55.

21 Barberi 1988, 19; Barberi 2001, 52.

22 Barberi 1988, 23; Baberi 2001, 50.

23 Siehe hierzu auch: Barberi 2001, 50-52.

24 Barberi 1988, 23; Barberi 2001, 50.
} 


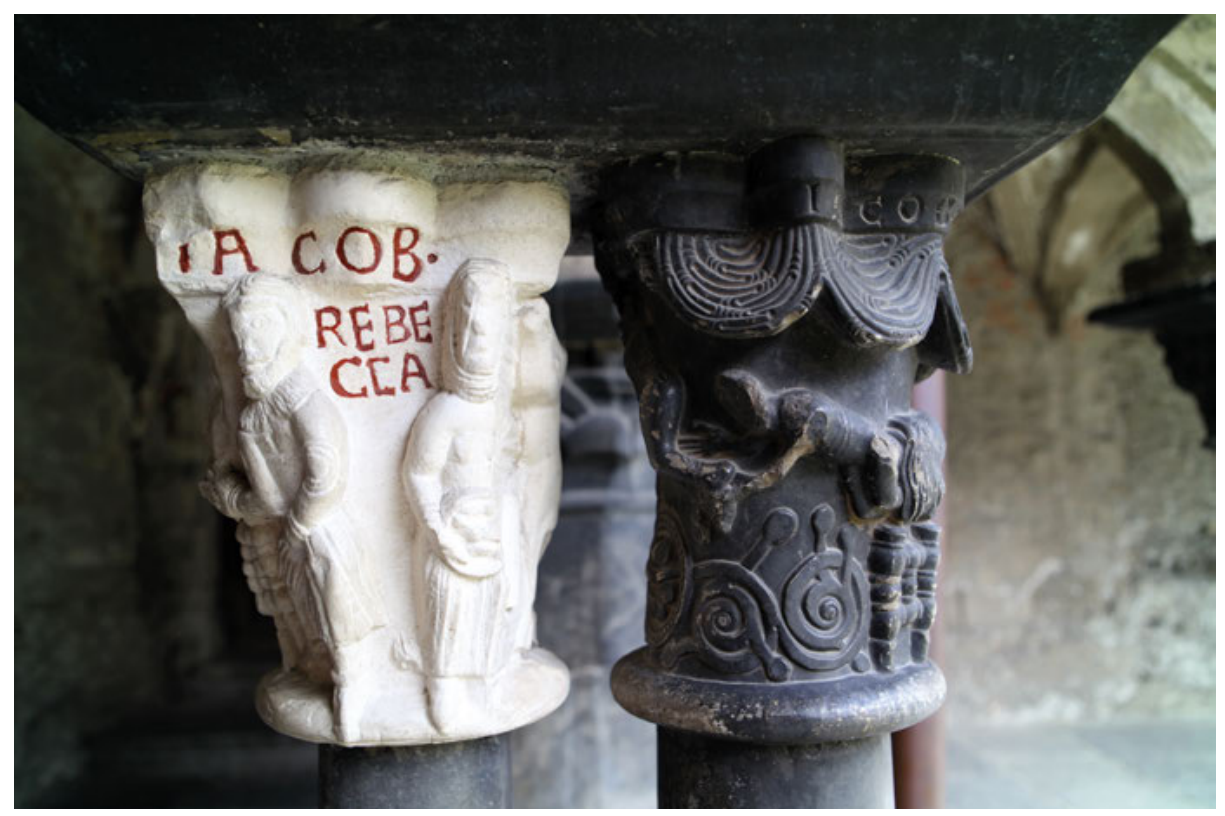

Abb. 2: Aosta, Santi Pietro ed Orso, Kreuzgang, Westflügel, Doppelkapitell Nr. 16, Südseite, Szenen aus dem Leben Jakobs.

den Quellen zufolge bereits im 2. Viertel des 17. Jahrhunderts der Fall. ${ }^{25}$ Nach dem Diebstahl eines Kapitells im Jahr 1997 wurde von der Denkmalpflege nach Fotos eine Kopie in Auftrag gegeben. Um dem heutigen Besucher einen Eindruck des ursprünglichen Zustands zu vermitteln, ${ }^{26}$ verzichtete man bei der Kopie auf die nachträgliche schwarze Fassung und hob die Inschriften mit roter Farbe hervor (Abb. 2).

Die ursprünglich mit roter Paste gefüllten Inschriften lassen sich z. B. am Kapitell mit der Gründungslegende im Südflügel belegen. Dort ist bei den Inschriften an einigen Stellen die schwarze Farbe abgeblättert. Darunter kam eine rötliche Farbpaste zum Vorschein mit der die Inschriften verfüllt waren (Abb. 3). ${ }^{27}$

Bevor auf die drei Kreuzgangflügel mit den romanischen Kapitellen eingegangen wird, muss auf eine allgemeine rezeptionspraktische Problematik bei Kapitellen in Kreuzgängen verwiesen werden. Man kann alle vier Seiten eines Kapitells mit ihren Darstellungen und ihren eventuell umlaufenden Inschriften nicht auf einmal wahrnehmen, da man nicht einfach um die Säule herumlaufen kann. Hierzu müsste man über das Bankett steigen, auf dem die Arkaden des Kreuzgangs stehen, oder immer

25 Barberi 2001, 50.

26 Barberi 2001, 52. Kapitell Nr.16. Den ursprünglichen Eindruck der weißen Marmorkapitelle kann man auch bei den im Zuge des Kreuzgangumbaus ausgebauten Kapitelle, die sich heute im Museo Civico di Torino befinden, nachvollziehen.

27 Siehe hierzu auch: Barberi 1988, 24; Barberi 2001, 50 u. Anm. 4. 


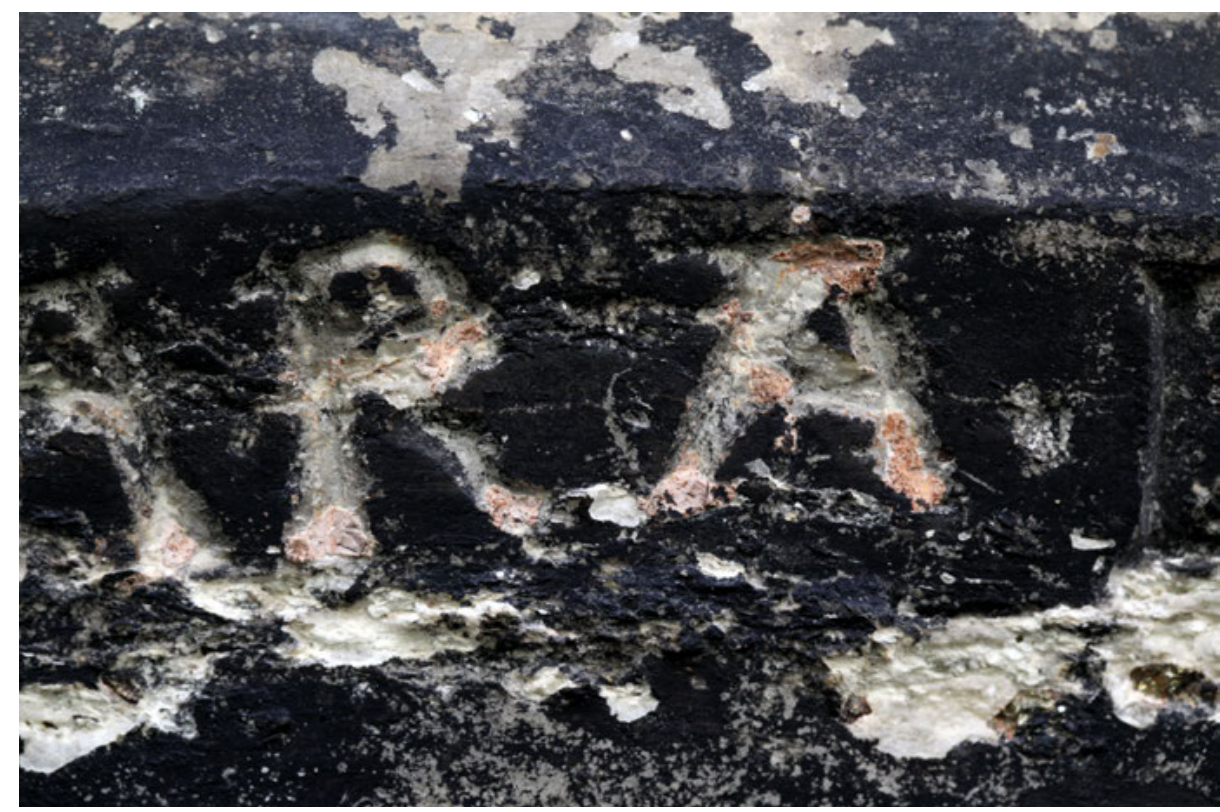

Abb. 3: Aosta, Santi Pietro ed Orso, Kreuzgang, Südflügel, Kapitell Nr. 32, Kapitell mit der Legende des heiligen Ursus, Nordseite, Inschrift, Fassungsreste.

wieder durch einen der Durchgänge in den Innenhof gehen und dann wieder zurücklaufen. Bei dem Kapitell, das die Legende mit dem heiligen Ursus illustriert und das oben auf der abgeschrägten Abakusplatte eine zweizeilige Umschrift und zudem Beischriften in den einzelnen Szenen aufweist, müsste man das Kapitell sogar mindestens zweimal umrunden. Daher sind die Darstellungen und Inschriften der Kapitelle, je nach Standpunkt des Betrachters, in ihrer Wahrnehmung und Sichtbarkeit und umlaufende Inschriften auch in ihrer Lesbarkeit eingeschränkt: Sie haben somit eine restringierte Präsenz, und zwar in räumlicher Hinsicht. ${ }^{28}$ Zudem ist, aufgrund ihrer Anbringung innerhalb eines Kreuzgangs, der nur oder jedenfalls zuallererst den Mitgliedern der Gemeinschaft zugänglich ist, auch eine Einschränkung auf personeller Ebene gegeben. ${ }^{29}$

An den Kapitellen des Nordflügels sind keine Inschriften vorhanden. Die Kapitelle sind durch alttestamentliche Szenen und Szenen aus der Kindheit Jesu, oder einfach vegetabil oder zoomorph geschmückt. Die Kapitelle des Westflügels sind historisierende Kapitelle, die die Geschichte des alttestamentlichen Patriarchen Jakobs darstellen (Abb. 2). Es wechseln sich immer Doppelkapitelle mit einfachen Kapitellen

28 Zur restringierten Schriftpräsenz siehe: Frese/Keil/Krüger 2014; Keil et al. 2019.

29 Zur restringier Präsenz von Inschriften auf personeller, zeitlicher und räumlicher Ebene siehe: Keil 2014. Für Stifterinschriften in gleicher Hinsicht siehe: Keil 2018. 
ab. Die Inschriften an den Kapitellen dienen nur zur Identifizierung des Abgebildeten. Es handelt sich also nur um Beischriften. ${ }^{30}$

Die meisten Inschriften befinden sich auf den Kapitellen des Südflügels. Das erste Kapitell von Westen ist ein Adlerkapitell, das keine Inschriften aufweist. Das folgende Kapitell ist mit Widderköpfen und Rankenwerk geschmückt. ${ }^{31}$ Die umlaufende Inschrift steht auf der Abakusplatte und beginnt auf der Südseite: MARMORIBVS . VARIIS · HEC · / EST · DISTINCTA · DECENTER • / FABRICA · NEC · MINVS · EST • DISPO/SITA CONVENIENTER $\cdot .^{32} \mathrm{Zu}$ dieser Inschrift gibt es in der Kirche selbst ein Pendant. 1999 wurde unter dem Fußboden des Kanonikerchors ein Fußbodenmosaik aufgedeckt. ${ }^{33}$ Es handelt sich um ein quadratisches Feld, in das ein Kreis eingeschrieben ist. In den Zwickeln sind Tiere und ein Hybridwesen dargestellt. Im Kreis sind mehrere Kreise so angeordnet, dass runde Bänder entstehen. Innen ist ein Mann auf einem Löwen sitzend dargestellt, der gerade den Rachen des Löwen aufreißt. Da der Mann lange Haare hat, dürfte es sich nach der Darstellungstradition um Samson handeln. ${ }^{34}$ Dann folgt eine Inschrift, die oben und unten mit kleinen Dreiecken verziert ist. Der Text ist ein bekanntes Palindrom aus der Antike: ROTAS OPERA TENET AREPO SATOR. ${ }^{35}$ Danach folgt ein Band mit Knotenmuster und zum Abschuss wieder eine Inschrift: + INTERIVS DOMINI · DOMVS · HEC · ORNATA · DECENTER - + QVERIT · EOS · QUI - SEMPER - EI · PSALLANT · REVERENTER $\cdot .^{36}$ Die Inschrift ist zweigeteilt, wobei der erste und zweite Teil jeweils die Hälfte des runden Bandes einnehmen. Der zweite Teil der Inschrift ist gespiegelt geschrieben. Die Inschrift thematisiert sowohl die Dekoration als auch den Gesang. ${ }^{37}$ Lucy Donkin hat dies mit der Sichtweise der Chorherren und ihrem Status in Verbindung gebracht, denen die aktuelle Debatte über Kunst und Psalmengesang bekannt gewesen sei. ${ }^{38}$ In einer anonymen Auslegung der Augustinusregel mit dem Titel Expositio in regulam beati Augustini, die wohl in der ersten Hälfte des 12. Jahrhunderts entstand, wird unter den Aktivitäten

$30 \mathrm{Zu}$ Beischriften siehe: Feraudi-Gruénais 2017.

31 Kapitell Nr. 25. Die Nummerierung der Kapitelle erfolgt nach Robert Berton und Sandra Barberi. Siehe die Pläne bei: Berton 1956, 11; Barberi 1988, 101.

32 „Mit verschiedenem Marmor ist dies anständig unterschieden. Die Kunst ist auch nicht weniger angemessen verteilt“. Das M und N von MINVS sind Unziale.

33 Zu dem Mosaik siehe: Papone/Vallet 2001; Perinetti 2005; Donkin 2008. Die Kirche war auch mit Wandmalereien geschmückt. Diese sind in Teilen wieder freigelegt worden. $\mathrm{Zu}$ den romanischen Wandmalereien siehe: Segre Montel 2001.

34 Siehe hierzu auch: Papone/Vallet 2001, 36f.

$35 \mathrm{Zu}$ den antiken Sator-Inschriften siehe: Hofmann 1978.

36 „Dieses Haus des Herrn ist im Inneren anständig geschmückt. Er sucht diejenigen, die ihm immer ehrfürchtig vorsingen“.

37 Lucy Donkin setzt den Gesang bereits in ihrer freien Übersetzung mit Psalmengesang gleich. Siehe: Donkin 2008, 75. Sie argumentiert dies später mit der Ähnlichkeit von Psalm 25, 8: Domine dilexi decorum domus tuae et locum habitationis gloriae tuae, und mit der Verwendung des Verbs psallare. Siehe: Donkin 2008, 83f.

38 Donkin 2008, 75. 
der Regularkanoniker das Darbringen von Psalmen, Hymnen und spirituellen Gesängen betont. ${ }^{39}$ Für Sandra Barberi hingegen sind Inschriften dieser Art ein stereotypes Formular, das die Kunst lobt und an verschiedenen Monumenten des 11. und 12. Jahrhunderts zu finden ist. ${ }^{40}$ In beiden Inschriften, Kapitell und Mosaik, wird von „anständigem Schmuck“ geschrieben. Vom Inhalt her vermutet Lucy Donkin, dass beide Inschriften ungefähr zur gleichen Zeit entstanden sind. ${ }^{41}$ Charles Bonnet und Renato Perinetti haben dies bereits vor ihr so gesehen und datieren beide Bildwerke in das fünfte Jahrzehnt des 12. Jahrhunderts. ${ }^{42}$ In der Augustinusregel steht nur im allegorischen Sinn etwas über die Ausschmückung der Ecclesia als Gemeinschaft der Gläubigen, aber nicht als Kirchenbau. Zur Ausschmückung der Kirche gibt es aber Textpassagen in Schriften von Augustinerchorherren. Diese stehen dem Schmuck jedoch meistens ablehnend gegenüber. Deswegen wurden bei den Inschriften die Worte ,anständig“ bzw. „angemessen geschmückt“ verwendet. ${ }^{43}$

Das nächste Kapitell hat keine Inschrift und zeigt Atlanten bzw. Eckfiguren mit in Vasen befindlichen Pflanzen, an denen sich diese festhalten.

Nach einem Durchgang vom Kreuzgang in den Kreuzgarten folgen fünf Kapitelle, die auf jeder Seite in einem Medaillon einen mit Nimbus versehenen bärtigen Propheten als Bruststück zeigen. Die Propheten halten in ihren Händen Rotuli. Alle Propheten sind auf dem Medaillon mit Namen bezeichnet. Auf dem Rotulus steht ein Teil eines Verses des dargestellten Propheten, der häufig auf den Medaillons fortgesetzt wird. Die Kapitelle werden ausgehend von der Südseite beschrieben, einmal da dies die Sicht vom Kreuzgang aus ist und zum anderen, da diese Anordnung der Abfolge der Prophetenbücher in der Bibel entspricht. Im Anschluss an die Südseite wird entgegen des Uhrzeigersinns weiter vorangegangen, entsprechend der Leserichtung bei den anderen Kapitellen mit umlaufenden Inschriften.

Auf dem ersten Prophetenkapitell sind die letzten vier Propheten (Malachi, Sofonias, Aggeus, Zaccharias) aus dem Zwölfprophetenbuch dargestellt. ${ }^{44}$ Da das Kapitell im Süden mit Malachias, dem zwölften der zwölf kleinen Propheten beginnt, könnte das Kapitell bei einem Wiedereinbau um 90 Grad verdreht worden sein. Eigentlich

39 Donkin 2008, 84f. In psalmis, hymnis et canticis spiritualibus coelesti Domino deservire. Siehe: „Exposito“ (1854), 885A-B. Der Text wird in der Quellenausgabe noch Hugo von Sankt-Viktor zugeschrieben.

40 Barberi 2001, 49.

41 Donkin 2008, 78.

42 Bonnet/Perinetti 2001, 23.

43 Donkin 2008, 80f. Zur Augustinerregel siehe: Balthasar 1980, 158-171. Auf die Bildkritik vom Zisterziensermönch Bernhard von Clairvaux, die sich primär gegen Bilder im monastischen Bereich richtete und die Unterschiede zu den Bildkritiken der Augustinerchorherren kann hier nicht eingegangen werden. Zur Bildkritik Bernhards siehe: Frese 2006.

44 Kapitell Nr. 27. Die Namen der Propheten und die Bibelbelege entstammen der Vulgata. Alle Inschriften sind in romanischer Majuskel geschrieben. Auf die nicht in Kapitalis geschriebenen Buchstaben wird in den Fußnoten hingewiesen. 
müsste, wenn man die meisten anderen Kapitelle von der Anordnung her betrachtet, die heutige Ostseite ursprünglich nach Süden gerichtet gewesen sein, um das Kapitell mit dem neunten der zwölf Propheten beginnen zu lassen. An den Kapitellecken befinden sich vegetabile Formen und Vögel. Oben am Medaillon auf der Südseite steht MALA/CHIAS $\cdot .{ }^{45}$ Der Vers beginnt auf dem Schriftband: MALEDICTVS.${ }^{46}$ und wird unten auf dem Medaillon weitergeführt: DOLOSVS $\cdot .^{47}$ Es handelt sich um den Vers Malachias 1,14, der das Darbringen von falschen Opfern (geraubte, lahme oder kranke Tiere) thematisiert, was zur Verfluchung der Betrüger führt. Auf der Ostseite ist oben der Prophet SOPHO/NIAS · benannt. ${ }^{48}$ Auf dem Spruchband beginnt der Vers mit LAVDA · FILIA, der unten auf dem Medaillon mit SVON • (für Sion) ${ }^{49}$ weitergeführt wird..$^{50}$ Den Zusammenhang verdeutlicht Sofonias 3,14: Israel kann sich freuen, da der Herr Israel befreit hat, indem er die Machthaber beseitigt hat, und da er nun mitten unter ihnen weilt. Auf der Nordseite beginnt der Vers auf dem Rotulus mit EGO - MOVEBO - und unten auf dem Medaillon steht: AGGEVS : CELVUM : ${ }^{51}$ Hier wird also der Spruch nicht zusammenhängend geschrieben, sondern durch den Namen des Propheten geteilt. In den Versen um Aggeus 2,22 wird dieser vom Herrn aufgefordert, zum Statthalter von Juda zu gehen und diesem auszurichten, dass er den Himmel bewegen werde. Dies stellt die Verheißung der Befreiung dar. Auf der Westseite ist Zaccharias zu sehen, der ausnahmsweise wie sonst nur AGGEVS unten am Medaillon bezeichnet wird: + ZACHARIAS : (Abb.4). Auf dem Spruchband beginnt der Vers: IRATVS $\cdot \mathrm{E}(\mathrm{ST}) \cdot \mathrm{D}(\mathrm{OMI}) \mathrm{N}(\mathrm{V}) \mathrm{S},{ }^{52}$ der oben am Medaillon weitergeführt

45 Zwischen MALA und CHIAS ist eine Lücke gelassen, in der der Nimbus ist. Diese Lücke wird in der Transkription durch einen Schrägbalken angezeigt. In dieser Lücke wurde bei Malachias nachträglich das Wort LONGIS eingeritzt.

46 Das $\mathrm{A}$ und $\mathrm{L}$ und $\mathrm{T}$ und $\mathrm{V}$ bilden jeweils Ligaturen.

47 Übersetzung der Inschrift: „Malachias: Verflucht ist der Betrüger“. Vulgatatext: Mal 1,14: maledictus dolosus qui habet in grege suo masculum et votum faciens immolat debile Domino quia rex magnus ego dicit Dominus exercituum et nomen meum horribile in gentibus.

48 Das $h$ ist ein unziales $\mathrm{H}$.

49 Statt ein I steht in SVON ein V.

50 Übersetzung der Inschrift: „Sofonias: Jubel, Tocher Zion“. Vulgatatext: So 3,14: lauda filia Sion iubilate Israhel laetare et exulta in omni corde filia Hierusalem. „lauda“ oder/und „exulta“ „filia Sion“ ist auch an Stellen anderer Propheten zu finden: Za 9,9: exulta satis filia Sion iubila filia Hierusalem ecce rex tuus veniet tibi iustus et salvator ipse pauper et ascendens super asinum et super pullum filiam asinae; Za 2,10 (14): lauda et laetare filia Sion quia ecce ego venio et habitabo in medio tui at Dominus; Is 12,6: exulta et lauda habitatio Sion quia magnus in medio tui Sanctus Israhel.

51 Übersetzung der Inschrift: „Ich bewege den Himmel“. Vulgatatext: Agg 2,22: loquere ad Zorobabel ducem Iuda dicens, ego movebo caelum pariter et terram. Bei Aggeus selbst gibt es noch eine Stelle, die nahezu gleich lautet: Agg 2,7: quia haec dicit Dominus exercituum adhuc unum modicum est et ego commovebo caelum et terram et mare et aridam.

52 Das T und V von IRATVS bilden eine Ligatur. Oberhalb des E von EST steht über die ganze Länge ein waagrechter Strich zur Kennzeichnung der Suspensionskürzung. Das D und N von DOMINVS bilden eine Ligatur. 


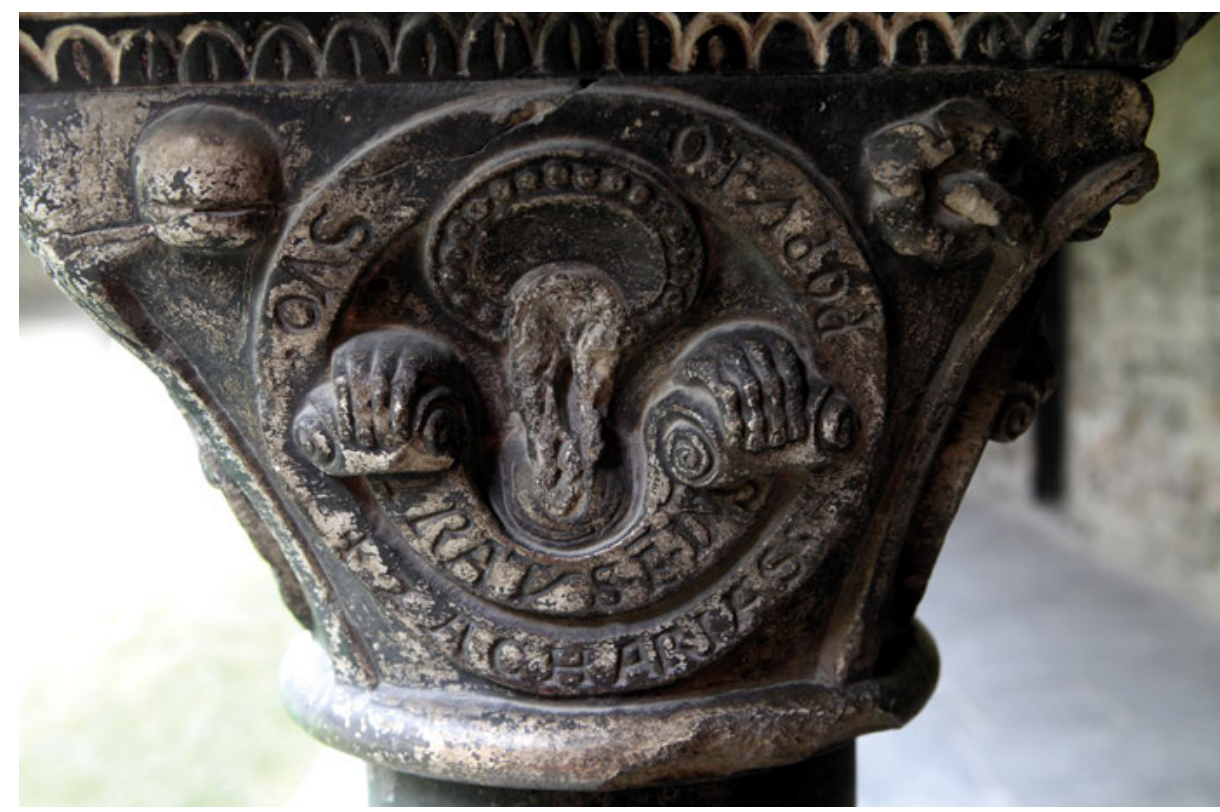

Abb. 4: Aosta, Santi Pietro ed Orso, Kreuzgang, Südflügel, Kapitell Nr. 27, Westseite, Prophet Zacharias.

wird: · SVO · POPVLO. ${ }^{53}$ Das SVO POPVLO steht auf dem Kopf und die Leserichtung scheint von rechts nach links zu gehen. Die Inschrift verläuft also im Rund des Medaillons weiter. Daher scheint der obere Teil für einen Betrachter von oben geschaffen zu sein. Das gespiegelte L lässt vermuten, dass der Stein beim Behauen nicht gedreht wurde, sondern die Inschrift verkehrt herum eingehauen wurde. Vielleicht ist diese besondere Anordnung hier bewusst gewählt worden, damit auffällt, dass bei diesem Medaillon etwas anders ist als bei den übrigen. Die Textpassage stammt nämlich nicht von Zaccharias, sondern gibt einen Teil des Psalms 105 (106),40 wieder. ${ }^{54}$ Der Herr ist wegen Israels Schuld durch ihre Sünden und den Götzendienst erzürnt. Im Anschluss daran zeigt er aber Erbarmen.

Am nächsten Kapitell sind die vier Propheten aus den Prophetenbüchern (Isaia, Hieeremia, Hiezechiel, Danhiel) wiedergegeben. ${ }^{55}$ Die Reihenfolge gleicht der in der Bibel, wenn man im Süden mit Isaia beginnt. Auf der Südseite steht oben am

53 Die Inschrift verläuft auf dem Kopf von links nach rechts. Das L von POPULO ist dabei gespiegelt. 54 Übersetzung der Inschrift: „Zaccharias: Der Herr ist gegen sein Volk aufgebracht“. Vulgatatext: Ps 105, 40: et iratus est furore Dominus in populo suo et abominatus est hereditatem suam. Der Vers entspricht auch dem Anfang von Is 5,25: ideo iratus est furor Domini in populo suo et extendit manum suam super eum et percussit eum et conturbati sunt montes et facta sunt morticina eorum quasi stercus in medio platearum in omnibus his non est aversus furor eius sed adhuc manus eius extenta.

55 Kapitel Nr. 28. 
Medaillon der Name des Propheten: + YSA/YAS $\cdot{ }^{56}$ Auf dem Spruchband steht: EGREDIETUR $\cdot{ }^{57}$ Die zitierte Stelle bei Isaia 26,21 spricht vom Jüngsten Gericht, zu dem der Herr seine himmlische Stätte verlässt. Auf der Ostseite ist Jeremias dargestellt. Er wird oben am Medaillon mit HI + hIERE/MIAS : bezeichnet. ${ }^{58}$ Der kurze Vers steht vollständig auf dem Spruchband: hIC : EST : DEVS : NOST(ER) ${ }^{59}$ In der Textstelle Ieremias 3,22 kommen die reuigen Sünder, die Söhne des Volkes Israel, zum Herrn. Sie sind zur Erkenntnis gelangt und zeigen Reue. Auf der Nordseite steht oben am Medaillon der Name des Propheten: + IEZE/ChIEL ${ }^{60}$ und der Vers beginnt auf dem Rotulus mit PADRES - COMEDE $^{61}$ und wird unten am Medaillon weitergeführt mit RVNT • VVAM - ACERBAM : (Abb. 5). ${ }^{62}$ In den Versen um Hiezechiel 18,2 fragt der Herr, warum es in Israel den Spruch gäbe, dass die Väter unreife Trauben äßen und den Söhnen die Zähne stumpf würden. Sie sollen diesen Spruch nicht mehr verwenden. Nur die Personen, die gesündigt haben (also die Väter und nicht die unschuldigen Söhne), sollen sterben. Auf der Westseite steht oben DANI/HEL •. Auf dem Spruchband steht: ASPICIEBAM - IN. Der Vers wird unten auf dem Medaillon fortgesetzt: VISV NOCTIS. ${ }^{63}$ Die Vision in Danhiel 2,13 ist eine Vision des Jüngsten Gerichts.

56 Über dem Y steht ein Punkt. Das zweite A hat links einen gebogenen Schaft der in einen gebogenen Deckbalken übergeht. Es handelt sich bei dem zweiten um ein pseudounziales A.

57 Das erste E ist eine Unziale, das zweite eine Kapitalis und das dritte wieder eine Unziale. Das G hat eine eingerollte Cauda. Das U ist ebenfalls eine Unziale. Übersetzung der Inschrift: Er wird ... verlassen. Vulgatatext: Is 26,21: ecce enim Dominus egreditur de loco suo ut visitet iniquitatem habitatoris terrae contra eum et revelabit terra sanguinem suum et non operiet ultra interfectos suos. Bei Is 11,1 steht die gleiche Verbform, aber der Inhalt mit der Wurzel Jesse passt nicht in den Gesamtzusammenhang der Kapitelle. Beim Propheten Ieremias findet sich die Verbform auch in gleichem Zusammenhang wie bei Is 26,21: Ier 25,32: haec dicit Dominus exercituum ecce adflictio egredietur de gente in gentem et turbo magnus egredietur a summitatibus terrae.

58 Das erste HI ist zwar eingehauen, aber es ist ohne jede Betonung und Zierde und unterscheidet sich so deutlich von den mittelalterlichen Inschriften. Es ist daher höchstwahrscheinlich erst später hinzugefügt worden. Das h ist eine Unziale. Die E sind auch unzial. Das M ist ebenfalls eine Unziale. Am Schluss des Namens stehen drei Punkte übereinander.

59 Das H und die E sind Unziale. Das N ist ein rundes N. Vom T ist nur der Schaft gut sichtbar. Die Worttrenner bestehen aus drei übereinander liegenden Punkten. Übersetzung der Inschrift: „Hier ist unser Gott“. Vulgatatext: Ier 3,22: convertimini filii revertentes et sanabo aversiones vestras ecce nos venimus ad te tu enim es Dominus Deus noster.

60 Die $\mathrm{E}$ und das $\mathrm{H}$ sind Unzialen.

61 Die ersten beiden E sind Kapitale und das dritte eine Unziale.

62 Zwischen RVNT und VVAM steht ein Zeichen, eine liegende Acht, an die links noch ein Halbkreis angesetzt ist. Übersetzung der Inschrift: „Die Väter haben saure (unreife) Trauben gegessen“. Vulgatatext: Ez 18,2: quid est quod inter vos parabolam vertitis in proverbium istud in terra Israhel dicentes patres comederunt uvam acerbam et dentes filiorum obstupescunt. Die Passage der Inschrift steht auch bei Ieronimus: Ier 31,29: in diebus illis non dicent ultra patres comederunt uvam acerbam et dentes filiorum obstipuerunt.

63 Der Abstand zwischen VISV und NOCTIS ist unüblich groß. Dies kann daran liegen, dass der Bogen des Medaillons sich unten verschmälert. Übersetzung der Inschrift: „Ich sah in einer Vision 


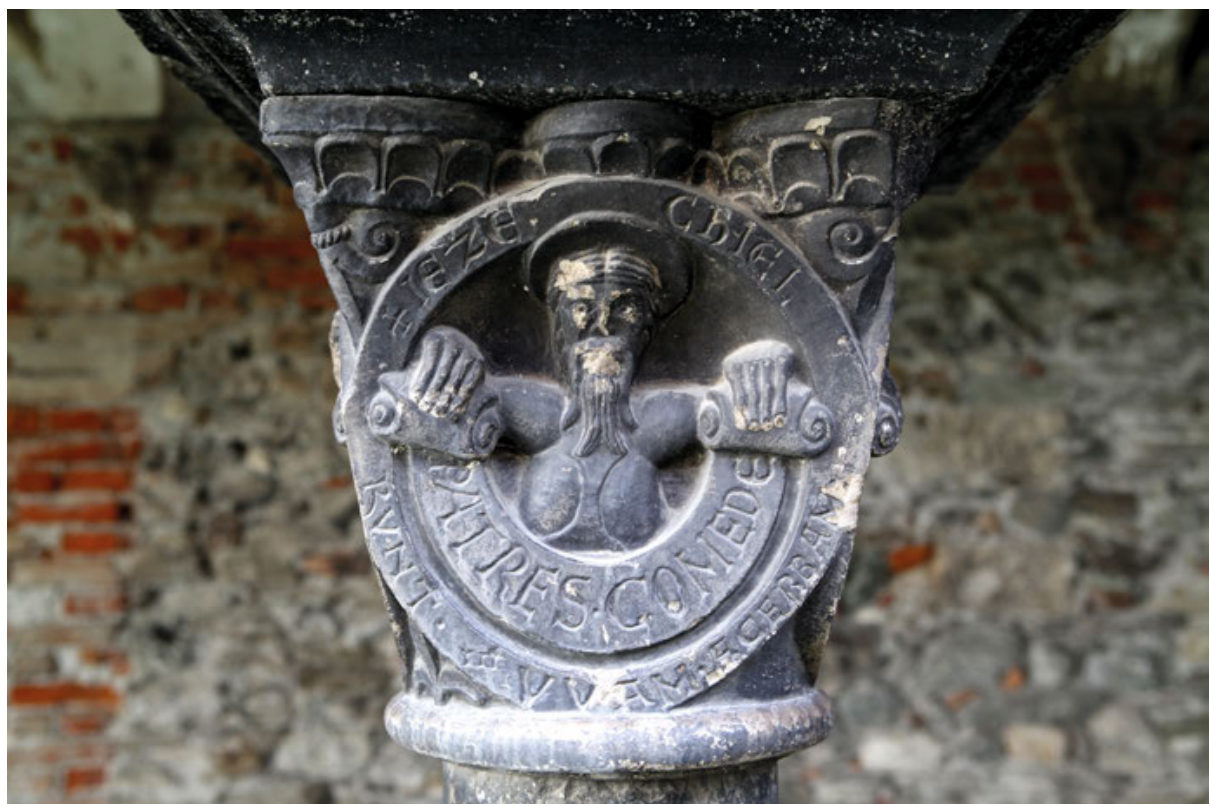

Abb. 5: Aosta, Santi Pietro ed Orso, Kreuzgang, Südflügel, Kapitell Nr. 28, Nordseite, Prophet Hiezechiel.

Danach folgen zwei Kapitelle mit Propheten aus dem Zwölfprophetenbuch. Zuerst sind die mittleren vier dargestellt (Abacuc, Iona, Micha, Naum). ${ }^{64}$ Da das Kapitell im Süden mit Abacuc, dem achten der zwölf kleinen Propheten beginnt, könnte das Kapitell bei einem Wiedereinbau um 90 Grad verdreht worden sein. Eigentlich müsste, wenn man die Anordnung der anderen Kapitelle betrachtet, die heutige Ostseite im Süden sein, um das Kapitell mit dem fünften der zwölf Propheten beginnen zu lassen. Oben am Medaillon auf der Südseite steht: ABA/CVC $\cdot$. Der Vers beginnt auf dem Spruchband: VE $\cdot$ QVI $\cdot$ POTV(M) - DAT ${ }^{65}$ und wird unten auf dem Medaillon weitergeführt: AMICO - SVO $\cdot{ }^{66}$ Durch die Passage um Abacuc 2,15 wird deutlich, dass man seinen Zorn nicht weitergeben soll, da man sich sonst um seine Ehre bringt. Auf der Ostseite steht oben am Medaillon mit ION/AS : der Name des

der Nacht“. Vulgatatext: Dn 7,13: aspiciebam ergo in visione noctis et ecce cum nubibus caeli quasi filius hominis veniebat et usque ad antiquum dierum pervenit et in conspectu eius obtulerunt eum.

64 Kapitell Nr. 29.

65 Das Q ist kleiner als alle anderen Buchstaben und der danach folgende Buchstabe, das V steht über der Cauda des Q. Das T und V bilden eine Ligatur. Am unteren Ende des Schaftes des T führt ein Strich nach schräg oben. Dies ist ein Kürzungszeichen.

66 Übersetzung der Inschrift: „Wehe dem, der seinem Freund das Getränk gibt“. Vulgatatext: Hab 2,15: vae qui potum dat amico suo mittens fel suum et inebrians ut aspiciat nuditatem eius. 


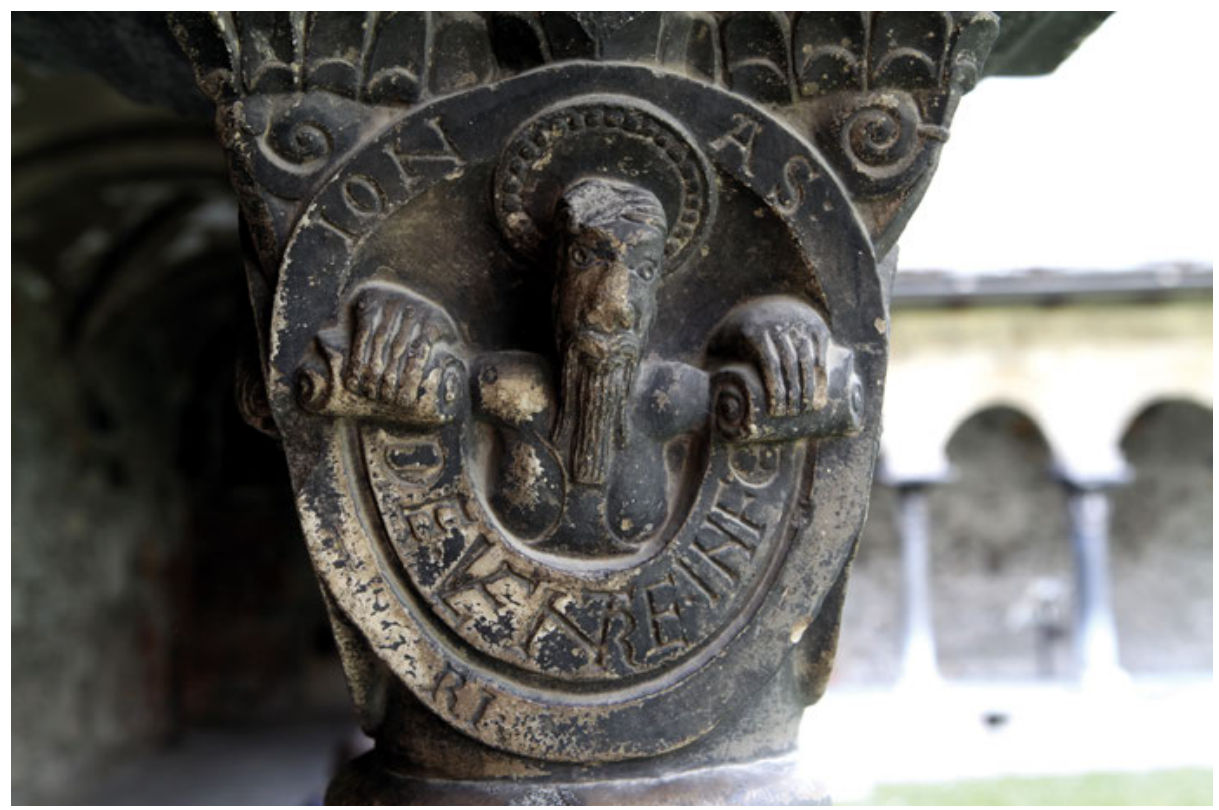

Abb. 6: Aosta, Santi Pietro ed Orso, Kreuzgang, Südflügel, Kapitell Nr. 29, Ostseite, Prophet lonas.

Propheten (Abb.6). Der Vers beginnt auf dem Rotulus mit DE - VENTRE - INFE ${ }^{67}$ und wird unten auf dem Medaillon weitergeführt mit der Endung RI $\cdot{ }^{68}$ In den Versen um Ionas 2,3 erfährt man, dass der Herr ihn erhört und befreit hat, indem er dem großen Fisch geboten hat, ihn auszuspeien. Auf der Nordseite steht oben auf dem Medaillon der Name MICh/EAS : ${ }^{69}$ und der Vers auf dem Rotulus beginnt mit PERIIT $\cdot$ S(AN)C(TV)S $\cdot$ DE, ${ }^{70}$ und endet unten auf dem Medaillon mit TERRA $:{ }^{71}$ In der Stelle um Micha 7,2 gehen die Frommen weg aus dem Land wegen all der Untreue im Staat und

67 Das V und E bilden eine Ligatur. Das N, T und R ein nexus litteratum. Es handelt sich um eine Zusammenfügung von drei Buchstaben. Das letzte E ist im Gegensatz zu den anderen eine Unziale. 68 Übersetzung der Inschrift: „Aus dem Bauch der Unterwelt“. Vulgatatext: Ion 2,3: et dixit clamavi de tribulatione mea ad Dominum et exaudivit me de ventre inferni clamavi et exaudisti vocem meam. 69 Das h ist eine Unziale. Nach dem Doppelpunkt am Ende des Namens folgt eine Schlangenlinie um den Platz auf dem Medaillonrand bis zum Beginn der Schriftrolle zu füllen.

70 Das zweite I ist dem T unterstellt. Das D besteht rechts aus einem geraden Schaft. Überleitend zum Bogen des D befinden sich gebrochene Bögen, die eindeutig auf eine spätere Entstehung hinweisen. Das DE scheint daher später hinzugefügt worden zu sein.

71 Das E ist eine Unziale. Bei dem A ist der linke Schrägschaft geschwungen und der rechte Schrägschaft nahezu gerade. Es handelt sich daher um ein pseudounziales A. Nach dem Doppelpunkt am Ende des Wortes folgt eine Schlangenlinie bis zum eingerollten Ende des Spruchbandes. Übersetzung der Inschrift: „Der Fromme verschwindet aus dem Land“. Vulgatatext: Mi 7,2: periit sanctus de terra et rectus in hominibus non est omnes in sanguine insidiantur vir fratrem suum venatur ad mortem. 


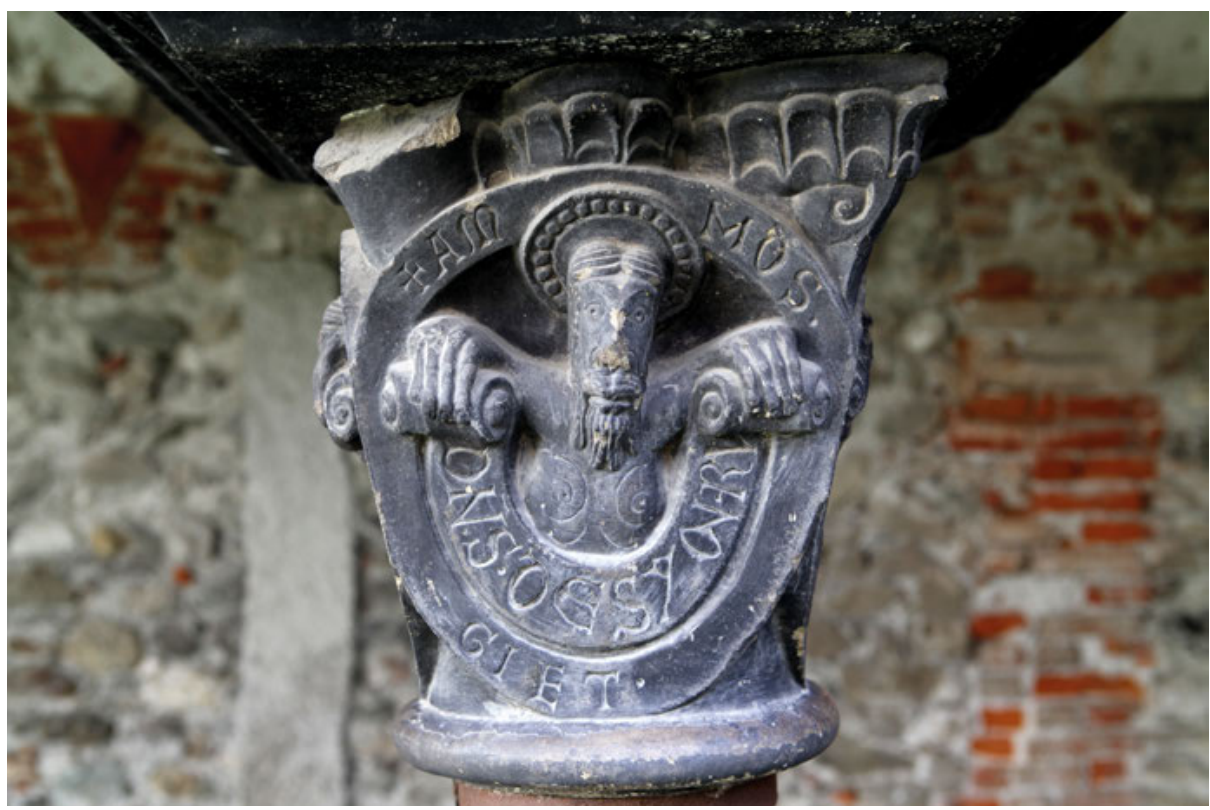

Abb.7: Aosta, Santi Pietro ed Orso, Kreuzgang, Südflügel, Kapitell Nr. 30, Nordseite, Prophet Amos.

in den Familien und hoffen auf den Herrn. Auf der Westseite steht oben NA/VM ${ }^{72}$ und auf dem Spruchband SOL · ORTVS · E(ST) $\cdot{ }^{73}$ Unten auf dem Medaillon ist eine Schlangenlinie eingehauen. Die Verse um Naum 3,17 beziehen sich auf Ninive. Die Wächter und Beamte sind nach Sonnenaufgang verschwunden. Die Herrschaft des Königs von Assur über die Stadt kann also nicht mehr ausgeführt werden.

Beim nächsten Kapitell sind die vier ersten Propheten aus dem Zwölfprophetenbuch wiedergegeben (Osee, Ioel, Amos, Abdias) ${ }^{74}$ Im Süden ist mit Osee der erste der Zwölfpropheten dargestellt und in Leserichtung die drei nächsten. Oben auf der Südseite steht der Name des Propheten + OSE/E :. ${ }^{75}$ Der Spruch beginnt auf dem Rotulus mit ET · ERIT · QVASI ${ }^{76}$ und wird unten auf dem Medaillon mit OLYUA · GL(ORI)A •

72 Das M ist eine Unziale. Vor dem Anfang des Namens beginnt eine Schlangenlinie, die um den ganzen restlichen Rand des Medaillons bis zum Ende des Namens verläuft.

73 Das $\mathrm{T}$ und V bilden eine Ligatur. Über dem E befindet sich über die gesamte Breite hinweg ein waagrechter Strich, der die Suspensionskürzung anzeigt. Übersetzung der Inschrift: „Die Sonne geht auf“. Vulgatatext: Na 3,17: custodes tui quasi lucustae et parvuli tui quasi lucustae lucustarum quae considunt in sepibus in die frigoris sol ortus est et avolaverunt et non est cognitus locus earum ubi fuerint.

74 Kapitell Nr. 30.

75 Das erste E ist eine Kapitale und das zweite eine Unziale. Am Ende des Namens liegen drei Punkte übereinander.

76 Das erste E ist eine Unziale. Das V und A von QVASI bilden eine Ligatur. 
EIVS • weitergeführt. ${ }^{77}$ In der Stelle um Osee 14,7 wird Israel aufgefordert nur dem Herrn zu huldigen. Oben auf dem Medaillon der Ostseite steht der Namen des Propheten: + IO/EL : ${ }^{78}$ Sein Vers befindet sich fast ganz auf dem Spruchband: PL(A)NGE QVASI ·VIRGO ${ }^{79}$ Unten auf dem Medaillon steht nur ein Buchstabe: A(CCINCTA). ${ }^{80}$ Nach Ioel 1,8 sollen die Ältesten über die Auswirkungen der Heuschreckenplage klagen. Auf der Nordseite wird oben der Prophet + AM/MOS $\cdot$ genannt (Abb.7). ${ }^{81}$ Das letzte Wort des im Rotulus mit D(OMI)N(V)S - DE SYON $\cdot \mathrm{RV}^{82}$ beginnenden Vers wird unten auf dem Medaillon mit GIET • beendet. ${ }^{83}$ In Amos 1,2 droht der Herr Israel und seinen Nachbarn. Der Herr verzeiht nicht, er fordert Sühne. Auf der Westseite steht oben auf dem Medaillon der Name des Propheten: + ABDI/AS $\cdot{ }^{84}$ Der Vers beginnt auf dem Spruchband: P(ER)DA(M) - SAPIENTES $\cdot \mathrm{DE}^{85}$ und wird unten auf dem Medaillon fortgesetzt: IDUMEA. ${ }^{86}$ In den Versen um Abdias 1,8 wird die Schuld

77 Oberhalb des Y ist ein Punkt. Das U ist nicht als V, sondern als U wiedergegeben, dessen linker Schaft gebogen ist und unten in einem Bogen zum rechten geraden Schaft überleitet. Das L ist durchgestrichen und hat oben einen kleinen Deckbalken. Dies sind Zeichen für eine Kontraktionskürzung. Übersetzung der Inschrift: „Und dessen Ruhm wird wie der des Olivenbaums sein“. Vulgatatext: Os 14,7: ibunt rami eius et erit quasi oliva gloria eius et odor eius ut Libani.

78 Am Ende des Namens stehen drei Punkte übereinander.

79 Für das A ist kein Kürzungszeichen zu erkennen. Das $\mathrm{G}$ hat eine eingerollte Cauda. Das Q und V von QVASI bilden eine Ligatur. Das V von VIRGO sieht aus wie ein U, in das das I eingestellt ist, da das $\mathrm{U}$ und das $\mathrm{R}$ eine Ligatur bilden.

80 Das alleine stehende A steht an einer Position, bei der man vermutet, dass noch weitere Buchstaben ausgeführt werden sollten. Da die Schrägschäfte des A unten breiter auslaufen als sonst üblich, könnte es sich auch um eine spätere Zutat handeln. Übersetzung der Inschrift: „Klage wie eine Jungfrau im Trauergewand“. Vulgatatext: Ioel 1, 8: plange quasi virgo accincta sacco super virum pubertatis suae.

81 Üblicherweise wird Amos nur mit einem M geschrieben, aber vielleicht wurden zwei M wegen der Trennung des Namens in zwei Hälften um den Nimbus gewählt. Interessanterweise ist das erste M eine Unziale und das zweite eine Kapitale.

82 Über dem N befindet sich ein kleiner Schrägstrich als Kürzungszeichen. Er ist anders als bei den anderen Kapitellen. Er ist deutlich kürzer und auch dünner. Es könnte sich daher auch um eine spätere Ergänzung handeln. Die beiden Buchstaben des DE sind Unziale. Das O und N von SYON bilden eine Ligatur.

83 Das G hat eine eingerollte Cauda. Übersetzung der Inschrift: „Der Herr brüllt vom Zion her“. Vulgatatext: Am 1,2: et dixit Dominus de Sion rugiet et de Hierusalem dabit vocem suam et luxuerunt speciosa pastorum et exsiccatus est vertex Carmeli. Die Passage der Inschrift ist auch bei Ioel wiedergegeben: Ioel 3, 16: et Dominus de Sion rugiet et de Hierusalem dabit vocem suam et movebuntur caeli et terra et Dominus spes populi sui et fortitudo filiorum Israhel.

84 Das erste A ist ein pseudounziales A. Der linke Schrägschaft ist geschwungen und geht in den Deckbalken über.

85 Der Schaft vom P ist durchgestrichen. Dies stellt ein Kürzungszeichen dar. Oberhalb des A ist ein waagrechter Strich, der ebenfalls ein Kürzungszeichen bildet. Das T und das E von SAPIENTES bilden eine Ligatur.

86 Übersetzung der Inschrift: „Ich vernichte die Weisen von Edom“. Vulgatatext: Abd 1,8: numquid non in die illa dicit Dominus perdam sapientes de Idumea et prudentiam de monte Esau. 


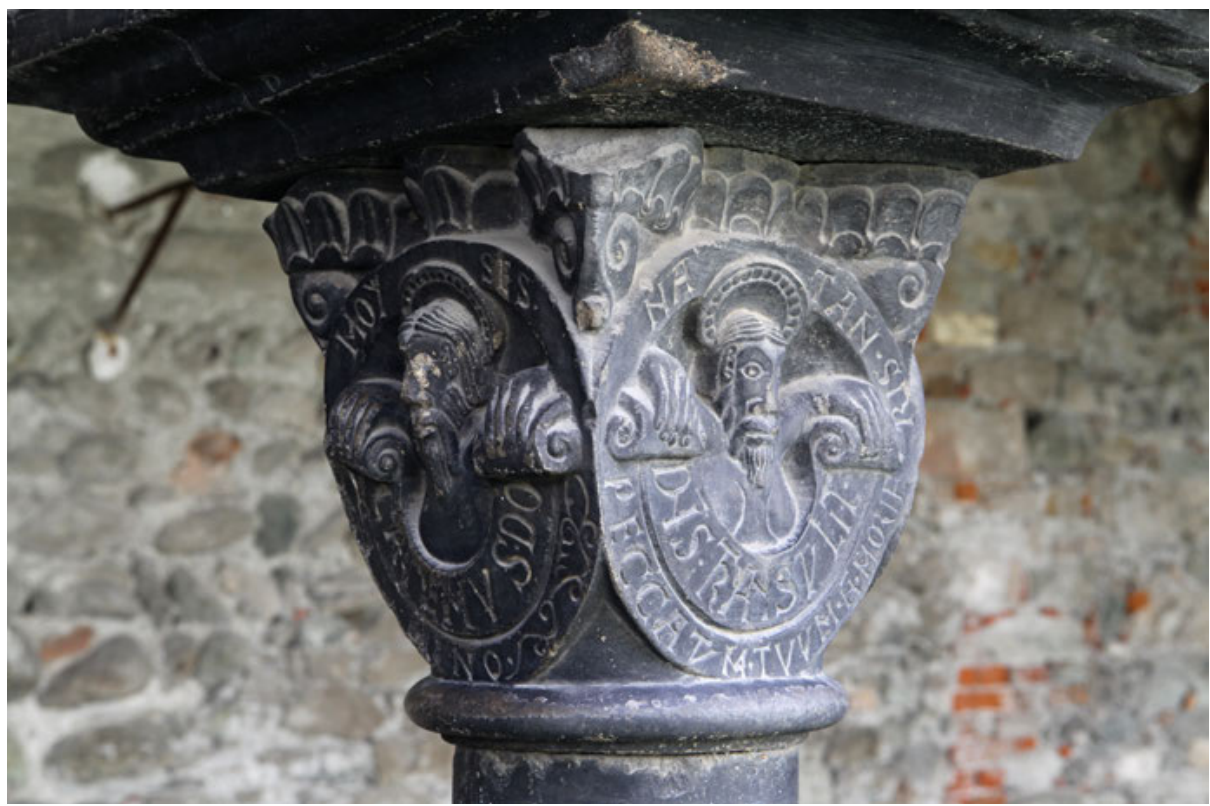

Abb. 8: Aosta, Santi Pietro ed Orso, Kreuzgang, Südflügel, Kapitell Nr. 31, Ost- und Nordseite, Prophet Moses und Prophet Natan.

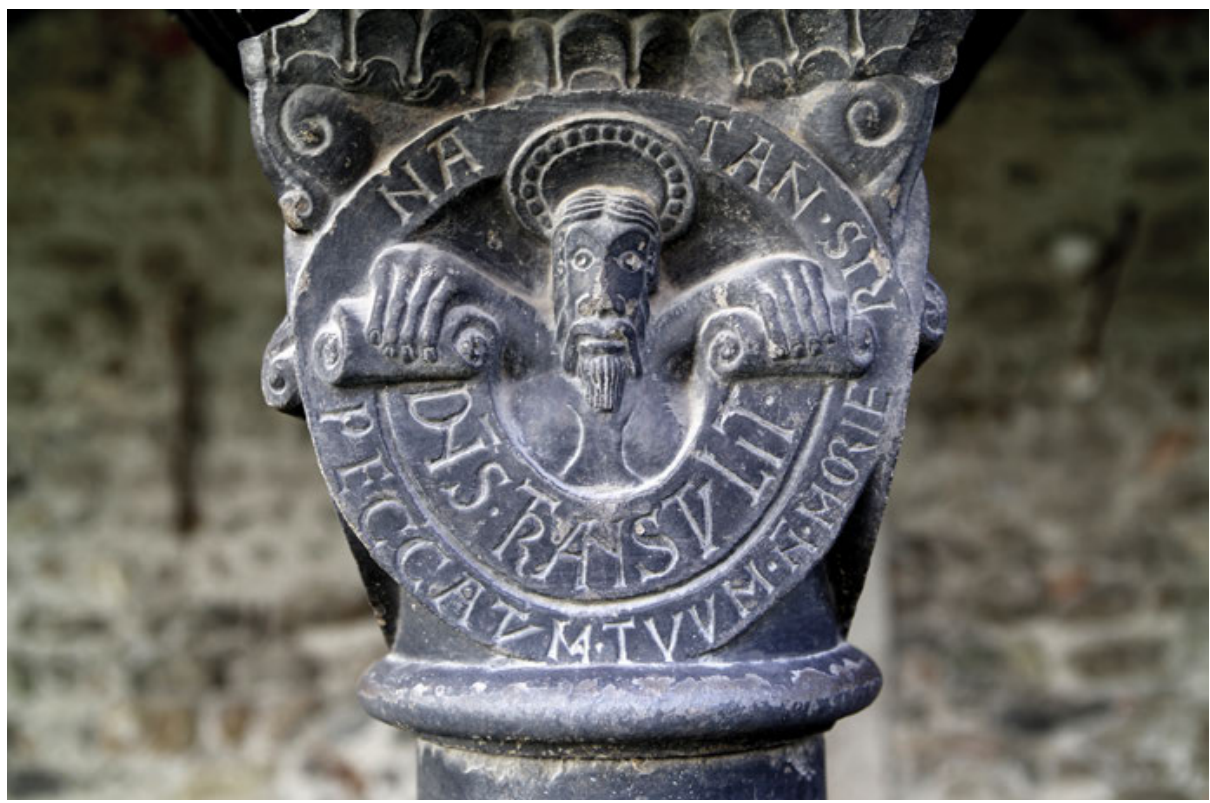

Abb. 9: Aosta, Santi Pietro ed Orso, Kreuzgang, Südflügel, Kapitell Nr. 31, Nordseite, Prophet Natan. 
Edoms aufgeführt und die Strafe angekündigt. Auf dem Berg Sion hingegen wird man gerettet werden.

Das letzte Prophetenkapitell zeigt vier Propheten, die nicht mit eigenen Schriften in der Bibel vertreten sind (Elias, Moses, Natan, Balaam). ${ }^{87}$ Oben am Medaillon auf der Südseite steht: hELY/AS :. ${ }^{88}$ Der Spruch auf dem Rotulus: FACIAMVS · hIC ${ }^{89}$ wird unten auf dem Medaillon fortgesetzt: TRIA - TABERNACVLA. ${ }^{90}$ Der Text aus Matthäus 17,4 ist kein Spruch des Propheten Elias, sondern ein Ausspruch von Petrus anlässlich der Verklärung Christi in dem er sagte, dass er drei Hütten bauen wolle, je eine für ihn, Moses und Elias. Auf der Ostseite wird oben auf dem Medaillon der Prophet MOY/SES . ${ }^{91}$ benannt (Abb. 8). Der Vers beginnt mit CANTEMVS $\cdot$ DO $^{92}$ auf dem Spruchband und wird unten auf dem Medaillon weitergeführt: MINO $\cdot .^{93}$ Unten links und rechts von MINO ist das Medaillonband geschmückt. Links ist eine Lilie unterhalb des eingerollten Ende des Schriftbandes eingehauen und rechts eine Wellenlinie mit zusätzlich eingerollten Ausläufern, die gemeinsam eine Blattranke darstellen sollen, die in einer Blüte endet. Der Versteil der Inschrift kommt sowohl in Exodus 15,1 als auch in 15,21 vor. An der ersten Stelle singen Moses und die Kinder Israels zu Ehren des Herrn, da er Rösser und Wagen der sie verfolgenden Ägypter ins Meer geworfen hatte. An der zweiten Stelle wiederholt Miriam den Gesang, nachdem der Pharao mit Wagen und Pferden im Meer versunken war. Auf der Nordseite des Medaillons steht mehr als nur der Name des Propheten: NA/TAN · SIR (Abb. 8, 9). Die drei Buchstaben RIS stehen auf dem Kopf. Sie sind die Endung des Bibelverses, der von der unteren Hälfte des Medaillons fortgeführt wird. Auf dem Spruchband beginnt dieser Vers mit D(OMI)N(I)S · TRANSTVLIT $\cdot 9^{94}$ Dieser wird unten auf dem Medaillon fortgeführt: PECCATVM $\cdot$ TVVM $\cdot$ N(ON) $\cdot$ MORIE/RIS $\cdot .^{95}$ Die Stelle bei 2 Samuel 12,13 sind die

87 Kapitell Nr. 31.

$88 \mathrm{~h}$ und $\mathrm{E}$ sind Unziale und über dem Y steht ein Punkt.

89 Das M von FACIAMVS und das h von hIC sind Unzialen.

90 Das E von TABERNACVLA ist eine Unziale. Übersetzung der Inschrift: „Wir machen hier drei Hütten“. Vulgatatext: Mt 17,4: respondens autem Petrus dixit ad Iesum Domine bonum est nos hic esse si vis faciamus hic tria tabernacula tibi unum et Mosi unum et Heliae unum.

91 Über dem Y steht ein Punkt.

92 Das A und $\mathrm{N}$ und das $\mathrm{T}$ und $\mathrm{E}$ in CANTEMVS bilden jeweils eine Ligatur.

93 Übersetzung der Inschrift: „Wir singen dem Herrn“. Vulgatatext: Ex 15,1: tunc cecinit Moses et filii Israhel carmen hoc Domino et dixerunt Cantemus Domino gloriose enim magnificatus est equum et ascensorem deiecit in mare. Die beiden Wörter der Inschrift kommen kurz später erneut vor: Ex 15,21: quibus praecinebat dicens cantemus Domino gloriose enim magnificatus est equum et ascensorem eius deiecit in mare.

94 Das D und N von DNS bilden eine Ligatur. Über dem N ist ein waagrechter Strich, der die Kürzungen anzeigt. Das T und R, A und N und T und V von TRANSTVLIT bilden jeweils eine Ligatur. Das I ist etwas kleiner und steht unter dem linken Deckbalkenende des $\mathrm{T}$.

95 Das T und V von PECCATVM bilden eine Ligatur. Über dem N zeigt ein waagrechter Strich die Suspensionskürzung an. Das O und R von MORIERIS bilden eine Ligatur. Das letzte Wort ist durch das eingerollte Ende des Schriftbands unterbrochen in die Teile MORIE und RIS. Das RIS steht oben 
Worte Natans. David zeigte Reue und sagte zu Natan, dass er gegen den Herrn gesündigt habe, woraufhin ihm Natan sagt, dass der Herr die Sünde vergibt und er nicht sterben müsse. Auf der Westseite wird oben am Medaillon der dargestellte Prophet mit BALA/AM : bezeichnet. ${ }^{96}$ Der Vers beginnt auf dem Rotulus mit MORIATVR . ANIMA $^{97}$ und wird unten am Medaillon mit MEA $\cdot$ MORTE $\cdot$ IVSTORVM $\cdot$ beendet. $^{98}$ Vor dem Vers Numeri 23,10 opferte Balac auf Geheiß Balaams für den Herrn. Balaam sollte für Balac mit dem Herrn sprechen, der ihn aber zurück zu Balac schickt. Daraufhin „segnet“ Balaam das Volk (Israel), das er eigentlich verfluchen sollte und möchte wie diese den Tod der Gerechten sterben.

Die Verse der Prophetenkapitelle betonen mehrere Themen: den Zorn Gottes aufgrund der Sünden des Volkes Israel; man soll keine Sünden begehen; die Strafen Gottes für die Unterdrücker und die Freude darüber; reuige Sünder haben Hoffnung auf Erbarmen; Hinweise auf das bevorstehende Jüngste Gericht. Die Themenkomplexe laufen auf ein belehrendes und moralisierendes Programm hinaus. Um dieses zu konzipieren, wurden von den einzelnen Propheten prägnante Textpassagen ausgewählt, die ohne Wiederholungen einen beziehungsreichen Gesamtzusammenhang zu bilden scheinen. Da einige Versteile auch von anderen Propheten bekannt sind, lässt sich ein hoch komplexes Programm mit vielschichtigen Verweisen auf einer zweiten Ebene vermuten, das aber vielleicht auch wegen des potentiellen Vertauschens und Verdrehens mancher Kapitelle bei Restaurierungen heute nicht mehr einfach zu erschließen ist. ${ }^{99}$

Das nächste Kapitell, das größer als alle andere ist, zeigt die Legende des Heiligen Ursus (Abb.10). ${ }^{100}$ Durch seine runde Grundform gibt es keine eigentlichen Schauseiten, sondern die einzelnen Szenen der Legende gehen ineinander über. Bei jeder Einzelszene gibt es Beischriften, die diese und die einzelnen Personen und teilweise die wichtigsten Objekte benennen. Auf diese Szenen und Beischriften kann in

nach dem Namen des Propheten. Übersetzung der Inschrift: „Der Herr hat dir die Sünde vergeben, du wirst nicht sterben“. Vulgatatext: II Sm 12,13: et dixit David ad Nathan peccavi Domino dixitque Nathan ad David Dominus quoque transtulit peccatum tuum non morieris.

96 Das mittlere A ist ein pseudounziales A. Der linke Schrägschaft ist geschwungen und geht in den Deckbalken des A über und der rechte Schrägschaft ist gerade. Die beiden A, die durch den Nimbus getrennt sind, werden jeweils in unterschiedlichen Schriftarten ausgeführt. Dies scheint eine Spielerei zu sein, da dies auch bei den Buchstabendoppelungen an anderen Kapitellen vorkommt.

97 Das $T$ und $V$ von MORIATVR ist eine Ligatur und das A und N von ANIMA ebenfalls.

98 Mit dem M von MEA und dem M von IVSTORVM sind das erste und das letzte $M$ auf der unteren Medaillonhälfte Unzialen. Übersetzung der Inschrift: „Meine Seele möchte den Tod der Gerechten sterben“. Vulgatatext: Nm 23,10: quis dinumerare possit pulverem Iacob et nosse numerum stirpis Israhel moriatur anima mea morte iustorum et fiant novissima mea horum similia.

99 An einigen Stellen konnte der Verfasser Zusammenhänge eines geplanten Systems vermuten. Hier ist nicht der Raum, um dies auszuführen.

100 Kapitell Nr. 32. Zur Legende und ihrer bewussten Darstellung im Zusammenhang mit der Übergabe der Augustinusregel an den Konvent siehe: Ferrari 2015, 121f, 137-148. 


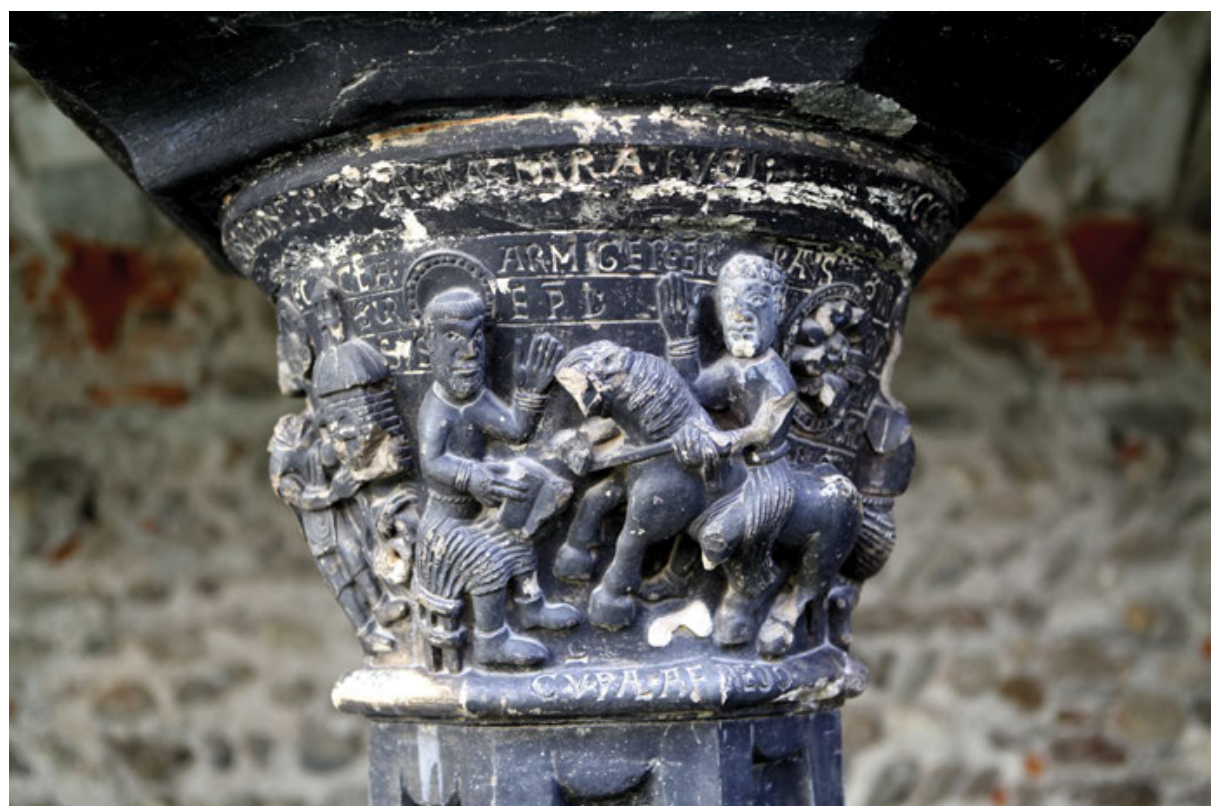

Abb. 10: Aosta, Santi Pietro ed Orso, Kreuzgang, Südflügel, Kapitell Nr. 32, Kapitell mit der Legende des heiligen Ursus, Nordseite.

diesem Zusammenhang nicht eingegangen werden. Oberhalb der Szenen verläuft auf der abgeschrägten Abakusplatte eine im Westen beginnende zweizeilige Inschrift (Abb.11):

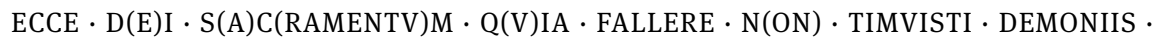

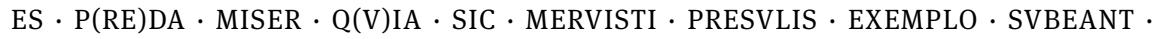
NIGRA $\cdot$ TARTARA $\cdot$ LVSI $\cdot$ QVI $\cdot$ NOS $\cdot$ I(TEM) $\cdot$ PVGNANT $\cdot$ CECA $\cdot$ FORMIDINE $\cdot$ FUSI. ${ }^{101}$ Sieh das Sakrament des Herrn: Weil du keine Angst hattest zu täuschen, bist du, Unglücklicher, die Beute von Dämonen, da du es so verdient hast. Das Beispiel des Bischofs zeigt, dass diejenigen die finstere Hölle erleiden, die uns ebenso durch Furcht getäuscht und getrieben, bekämpfen.

Die belehrende und ermahnende Inschrift betont das Dargestellte, indem in der Inschrift auf die am Kapitell wiedergegebenen Gräueltaten des Bischofs Ploceano Bezug genommen wird, und zudem das Programm der Prophetenkapitelle.

Danach folgen zwei Kapitelle mit Szenen aus dem Leben Jesu, die mit Beischriften versehen sind. Das darauf folgende Kapitell zeigt eine Szene aus der Geschichte des Konvents, nämlich die Übergabe der Ausgustinerregel, auf die bereits im Zusammen-

101 Kürzungszeichen in Form eines waagrechten Strichs sind auf dem I von DEI, dem C von SACRAMENTVM, dem N von NON und dem P von PREDA. Ligaturen sind das F und A von FALLERE, das N und $\mathrm{A}$ und $\mathrm{N}$ und $\mathrm{T}$ von PVGNANT und das $\mathrm{O}$ und $\mathrm{R}$ von FORMIDINE. Die M von TIMVISTI, DEMONIIS und FORMIDINE sind Unzialen. Bei den beiden QVIA ist das I über das Q gestellt. 


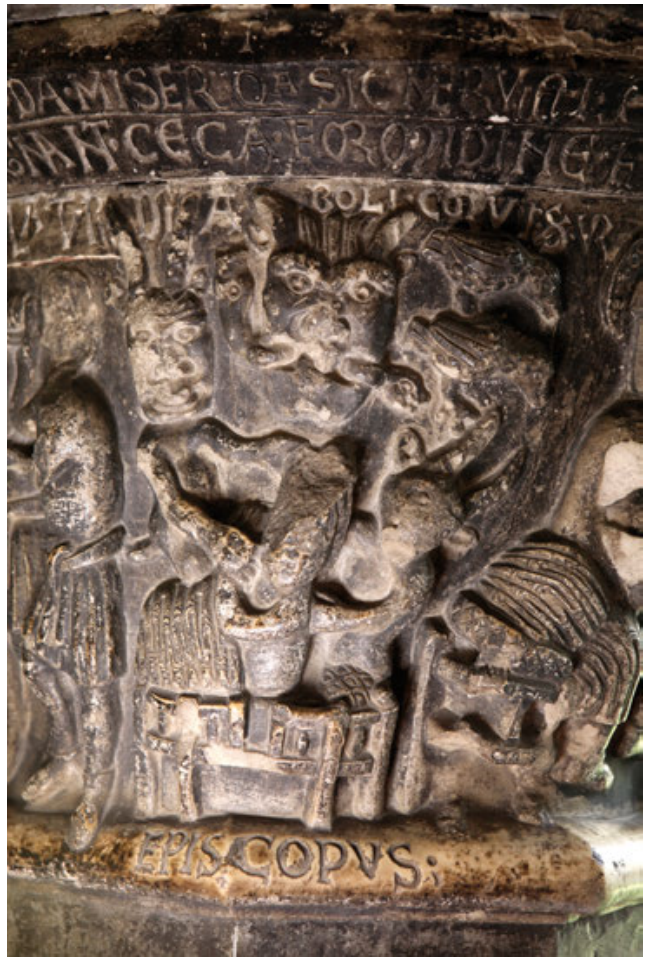

Abb. 11: Aosta, Santi Pietro ed Orso, Kreuzgang, Südflügel, Kapitell Nr. 32, Kapitell mit der Legende des heiligen Ursus, Südostseite.

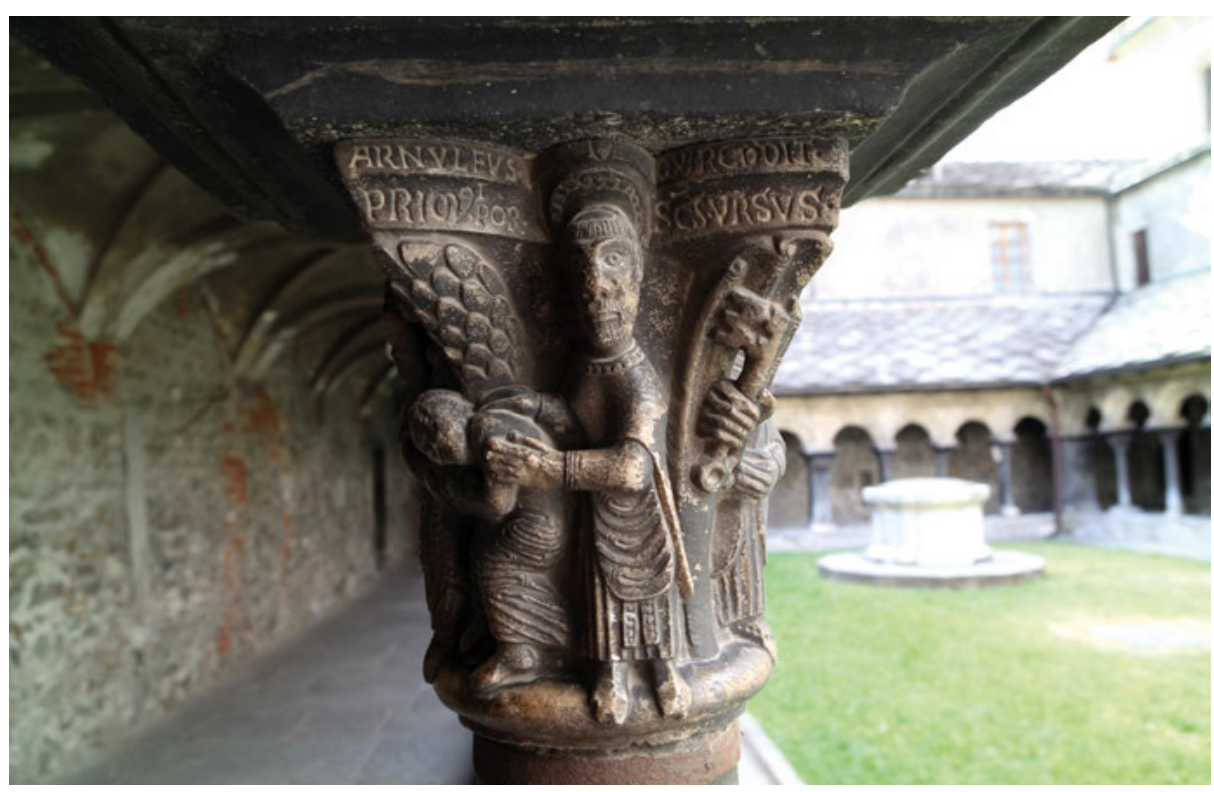

Abb. 12: Aosta, Santi Pietro ed Orso, Kreuzgang, Südflügel, Kapitell Nr. 35, Kapitell mit der Übergabe der Augustinerregel, Ostseite, Der heilige Ursus mit dem ersten Prior Arnulf von Avise. 


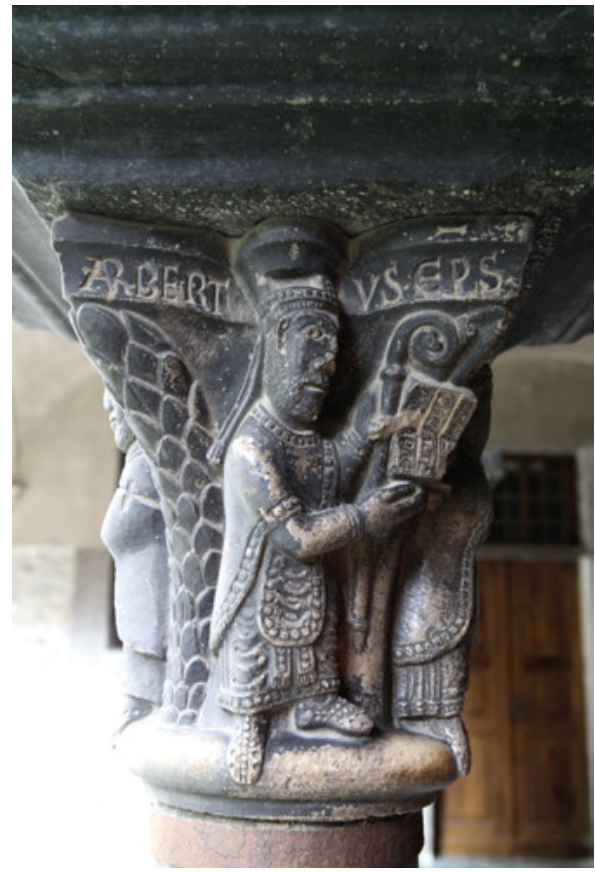

Abb.13: Aosta, Santi Pietro ed Orso, Kreuzgang, Südflügel, Kapitell Nr. 35, Kapitell mit der Übergabe der Augustinerregel, Westseite, Bischof Aribert von Aosta.

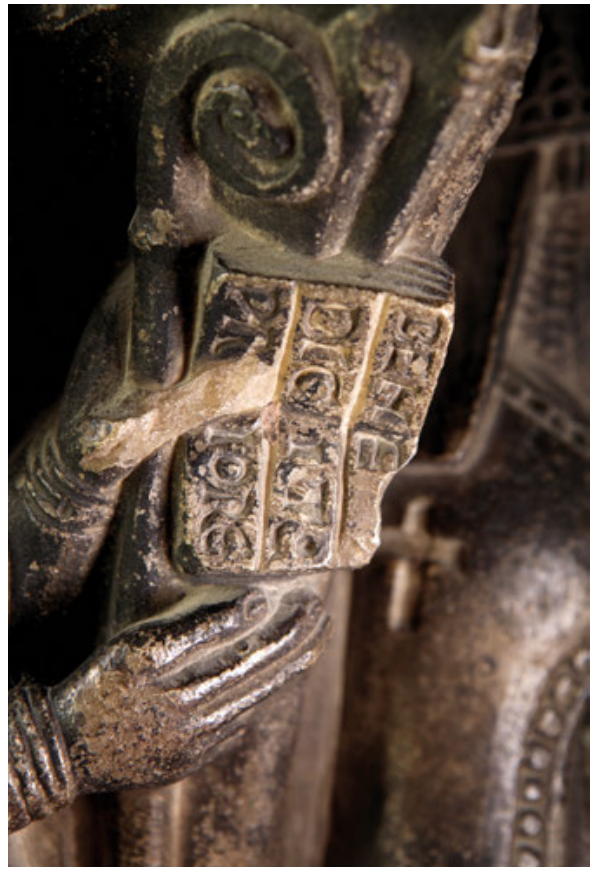

Abb.14: Aosta, Santi Pietro ed Orso, Kreuzgang, Südflügel, Kapitell Nr. 35, Kapitell mit der Übergabe der Augustinerregel, Westseite, Bischof Aribert von Aosta, Buch.

hang mit der Datierung eingegangen wurde. ${ }^{102}$ Die dargestellten Personen sind durch Beischriften mit Namen und Funktion bezeichnet. Auf der zum Kreuzgarten gerichtete Nordseite steht der Apostel Petrus mit zwei Schlüsseln in der Hand. Auf der Ostseite sieht man den heiligen Ursus stehend, wie er als Fürsprecher mit seinen Händen auf den Schultern des ersten Priors Arnulf von Avise, diesen in ehrfürchtiger Haltung zu Augustinus schiebt (Abb.12). Auf der Südseite hält der heilige Bischof Augustinus mit seiner rechten Hand, die demütig gefalteten Hände des ersten Priors Arnulf. Mit seiner linken Hand hält Augustinus einen Bischofsstab. Im Westen ist Aribert, der Bischof von Aosta mit Bischofsstab und einem Buch in der Hand dargestellt (Abb.13). Auf dem Buch steht eine Inschrift, die verdeutlicht, dass Augustinus den ersten Prior Arnulf segnet: BENE/DICIT(VR) • / PRIORE (Abb.14). Die Darstellung der Übergabe an die Augustinerchorherren ist nach Léon Pressouyre in einer Art repräsentiert, die er als ein Ergebnis der Kontroverse zwischen dem ordo monasticus und dem ordo regularis sieht, die im 11. und 12. Jahrhundert stattfand, in der beide Parteien für sich in Anspruch nahmen, dass ihre Art von Gemeinschaft der des apostolischen Modells

102 Kapitell Nr. 35. 


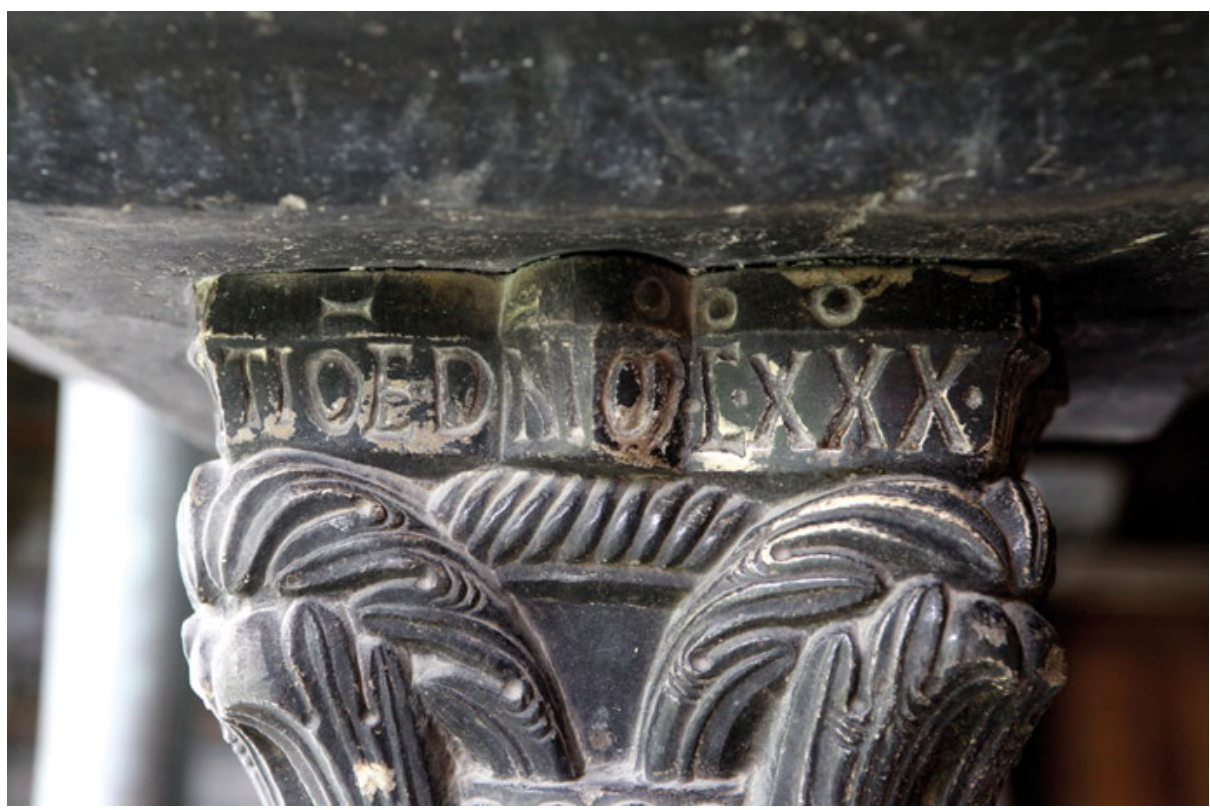

Abb.15: Aosta, Santi Pietro ed Orso, Kreuzgang, Südflügel, Kapitell Nr. 36, Kapitell mit der Gründungsinschrift, Westseite, Inschrift.

entspräche. ${ }^{103}$ Das Auftreten des Apostels Petrus ist hierbei entscheidend, da er an die Rolle des Papstes bei der Übergabe der Regel erinnert. ${ }^{104}$ Sandra Barberi sieht in Bischof Aribert den Entwerfer des Programms und ist der Meinung, dass er dem ersten Prior Arnulf bei der Organisation und Kontrolle der Arbeiten half. ${ }^{105}$ Das Kapitell, das den heute verkürzten Kreuzgangflügel abschließt, ist durch Blattwerk geschmückt. ${ }^{106}$ Auf der Abakusplatte steht die Gründungsinschrift des Augustinerchorherrenstiftes im Jahre 1133 bzw. nach heutiger Zeitrechnung 1132. Die umlaufende Inschrift beginnt auf der Nordseite (Abb.15): ANNO - AB INCARNA/TIO(N)E - D(OMI)NI M - C • $\mathrm{XXX} \cdot / \mathrm{III} \cdot \mathrm{IN} \mathrm{h}(\mathrm{OC}) \cdot \mathrm{CLAUSTRO} \cdot \mathrm{REGVLAR}(\mathrm{I}) \mathrm{S} / \cdot \operatorname{VITA} \cdot \mathrm{INCEPTA} \cdot \mathrm{EST} \cdot, \cdot{ }^{107} \mathrm{Die}$

103 Pressouyre 1973, 72-74.

104 Die Bedeutung dieser Rolle könnte auch ein Grund für das zweite Patrozinium San Pietro sein. Siehe hierzu: Ferrari 2015, $143 f$.

105 Barberi 1988, 30; Barberi 2001, 58.

106 Kapitell Nr.36.

107 „Im Jahr 1133 nach der Inkarnation des Herrn hat in diesem Kreuzgang das regulierte Leben begonnen“. Das N und A bei INCARNATIONE bilden eine Ligatur. Das dritte N fehlt und ist durch ein Kürzungszeichen über dem $\mathrm{O}$ angezeigt. Über dem N von DOMINI zeigt ebenfalls ein waagrechter Strich die Kontraktionskürzung an. Bei der Jahreszahl ist das M eine Unziale. Oberhalb des M, C und des mittleren X und I ist ein kleines O für die Kürzung der Ordnungszahlen angegeben. Das h von hOC ist eine Unziale und über diesem ist als Kürzungszeichen ein Punkt angegeben. Bei CLAUSTRO ist das A leicht hochgestellt über dem L und S und darüber nochmals das runde U. Das T und R bilden eine 
Inschrift ist im Zusammenhang mit dem daneben stehenden Übergabe-Kapitell zu betrachten. Hier wird noch einmal die Auswirkung der Übergabe der Augustinerregel verdeutlicht.

Auf der Nordseite des Kreuzgangs gibt es auf den Kapitellen keine Inschriften. Ob es im Ostflügel Inschriften gab, ist nicht bekannt. Zumindest die ausgebauten Kapitelle, die im Museo Civico di Torino erhalten sind, haben keine Inschriften. Im Westen sind die Szenen auf den Kapitellen nur mit Beischriften versehen. Im Südflügel sind die meisten Kapitelle sogar mit längeren Inschriften versehen. Es gibt drei Kapitelle mit umlaufenden Inschriften und die fünf Prophetenkapitelle mit ihren Spruchbändern.

Es fragt sich, wieso dies der Fall ist. Sind die Kapitelle etwa zu unterschiedlichen Zeiten entstanden und hat man sich vielleicht erst im Laufe des Baus entschieden, Kapitelle mit Inschriften anzufertigen? Oder gab es zwei Gruppen von Bildhauern, von denen eine Kapitelle ohne und eine mit Inschriften hergestellt hat? Oder stammen alle Kapitelle aus einer Bildhauerwerkstatt und hat der Auftraggeber bewusst entschieden, in einigen Flügeln Kapitelle mit und in anderen ohne Inschriften anzufertigen?

Um mögliche Antworten zu finden, muss man zunächst auf die Lebensweise der Augustinerchorherren eingehen. Im 11. und 12. Jahrhundert kam es zu einer Kanonikerreform, einer „Regulierung“ der Chorherrenstifte. Entscheidend war hierbei das Laterankonzil von 1059, bei der die Kleriker an ihre Lebensweise, die vita communis, das apostolische Leben, erinnert wurden. Regulierte Kanoniker sollten in einer gemeinschaftlichen Form leben und auf persönliches Eigentum verzichten. Ein Leben in der vita communis umfasst auch ein gemeinsames Schlafen im Dormitorium und ein gemeinsames Essen im Refektorium. Zuvor war dies bei Kanonikern nicht mehr der Fall gewesen. Am Anfang des 12. Jahrhunderts wurden Consuetudines, Regeln und Statuten, eingeführt, die nicht nur die Pflichten und Aufgaben der Kanoniker und deren Tagesablauf regelten, sondern auch andere Fragen, wie z. B. die Wahl des Priors. ${ }^{108}$ Die Consuetudines machen deutlich, dass nicht die monastische Askese entscheidend ist, sondern die apostolische Gemeinschaft. Ein Konvent eines Augustinerchorherrenstiftes machte also fast alles gemeinsam in unterschiedlichen gemeinschaftlichen Räumlichkeiten.

In der Augustinerregel sind wie in der Benediktinerregel, Lesungen beim Essen und eine abendliche Lesung vor dem Komplet bestimmt. ${ }^{109}$ Wo die Lesung vor dem Komplet stattfand ist nicht bekannt. Aus den Consuetudines der Augustinerchorherren

\footnotetext{
Ligatur und das O befindet sich über dem Worttrenner in Punktform. Das G von REGVLARIS ist ein eingerolltes $\mathrm{G}$ mit eingerollter Cauda. Das A und R bilden eine Ligatur und das I liegt wie ein waagrechtes Kürzungszeichen oberhalb des R. Bei VITA ist das I in das V eingestellt und das A ist etwas kleiner wiedergegeben.

108 Zur Kanonikerreform des 11. und 12. Jahrhunderts siehe: Heim 2003b, 137-144. Zu Augustinerchorherren siehe: Heim 2003a.
}

109 Untermann 2016, 92. 
in Rolduc (Klosterrath) wissen wir, dass es im Kreuzgang, wohl auf der Kirchenseite, entlang der Mauer Sitzbänke gab und dass Gegenüber an den Gittern zum Kreuzhof sich die Kanoniker rasierten und zur Fußwaschung Platz nahmen. Zudem wird vom Kreuzgang berichtet, dass es dort andere Bereiche gab, in denen der Konvent bei der Fußwaschung Psalmen sang oder die Novizen saßen und Unterricht erhalten haben. ${ }^{110}$

Lesegänge mit Sitzbänken waren in Kreuzgängen meistens im Norden an der Kirchenmauer untergebracht. ${ }^{111}$ Im Augustinerchorherrenstift auf dem Petersberg bei Halle stehen im Nordflügel die Pilaster an der Wand auf einer durchlaufenden Bank, die sicherlich als Sitzbank diente. ${ }^{112}$ Die Lage des Lesegangs im Norden wird auch praktische Gründe gehabt haben, da dort die Sonnenstrahlen einmal wärmen und vor allem genug Licht zum Lesen geben. Kam es in Aosta also bewusst zu einem Verzicht von Inschriften im Lesegang, damit die Kanoniker nicht durch Inschriften abgelenkt wurden? ${ }^{113}$

Nicht nur in den Consuetudines von Rolduc kommt eine Bezeichnung eines Gemeinschaftsraums vor, der in benediktinischen Konventen nicht existiert. ${ }^{114}$ Dieser Raum, der sich am Kreuzgang befindet, wird als conventus bezeichnet und dient den Kanonikern zum Lesen und Schreiben oder zum Warten bis sie durch die Glocke zum Chorgebet gerufen werden. ${ }^{115}$ Auch für das Augustinerchorherrenstift auf dem Petersberg bei Halle ist ein conventus belegt, den die Kanoniker für die abendliche Lesung benutzt haben. ${ }^{116}$ Aus den Consuetudines von Rolduc wissen wir auch, dass im conventus nicht gesprochen werden durfte und daher der Unterricht für die Novizen im Kreuzgang stattfand. Für Rolduc sind die sonst üblichen Räume um den Kreuzgang überliefert. Zudem wird betont, dass es neben dem Kapitelsaal ein auditorium geben soll, in dem man alles Notwendige besprechen kann. ${ }^{117}$

110 Untermann 2016, 94.

111 Da die Sitzbänke nur selten gemauert, sondern meistens aus Holz waren, sind sie in vielen Fällen nicht mehr nachweisbar. Siehe hierzu: Untermann 2009, $115 f$.

112 Krause 1967, 66.

113 Dieser Gedanke erinnert an die Aussage von Bernhard von Clairvaux, der in der Apologia von der Gefahr spricht, dass die Mönche in mit figürlichen Bauskulpturen geschmückten Kreuzgängen lieber in den mamora als in den codices lesen. Dabei sollen diese nicht die Artefakte bewundern, sondern über das Gesetz Gottes meditieren („in lege Dei meditando“). Siehe hierzu: Frese 2006, 31f. u. 100. In Aosta gibt es im Lesegang keine Inschriften, aber angemessenen Schmuck durch vegetabile und figürliche Bauskulptur. Es fragt sich, ob hier ein Einfluss des Zisterziensermönchs Bernhard von Clairvaux denkbar wäre oder eher einer durch die kritischen Schriften von Augustinerchorherren, die dem Schmuck ablehnend gegenüber standen.

114 Untermann 2016, 96.

115 Untermann 2016, 94.

116 Untermann 2016, 94.

117 Untermann 2016, 95. 
Schwierig ist es vor allem bei einem Kreuzgang wie bei SS. Pietro ed Orso in Aosta, bei dem nahezu keine ursprünglichen Anbauten überliefert sind, zu vermuten, wo sich die Räumlichkeiten ursprünglich befunden haben. ${ }^{118}$

An der Stelle des heutigen Durchgangs zum Kreuzgang sieht man in der Nordmauer eine vermauerte Pforte, die wohl in der zweiten Hälfte des 13. Jahrhunderts eingebaut wurde. Im Westflügel des Kreuzgangs sind in der Wand drei kleine Schachtfenster eingelassen, von denen eins eventuell aus dem 11. oder 12. und zwei eventuell aus dem 13. oder 14. Jahrhundert stammen. ${ }^{119}$ Das dahinter befindliche Gebäude wird heute „sagrestia delle messe“ bezeichnet und in das 11. oder 12. Jahrhundert datiert. ${ }^{120}$ Im Norden des Westflügels sieht man den vermauerten früheren Zugang zum Kreuzgang, ${ }^{121}$ der sich zwischen der Kirche und diesem Gebäude befand. Aus der Zeit der Entstehung des Kreuzgangs sind als anliegende Gebäude nur noch die Kirche und die sogenannte „sagrista delle Messe“ erhalten.

Die beiden Portale im Westen und Osten des Nordflügels, die in die Kirche führen, sind wohl an der gleichen Stelle eingebaut worden, an denen früher die Zugänge zur Kirche waren. ${ }^{122}$ Über die anderen Türen und die heute vermauerten Durchgänge lassen sich schwer Aussagen treffen. Allerdings könnte der vermauerte Durchgang in ungefähr der Mitte des Südflügels noch bauzeitlich sein.

Der Kreuzgang von SS. Pietro ad Orso in Aosta wurde also zwangsläufig häufig durch die Kanoniker aufgesucht und das nicht nur als Verkehrsweg, sondern auch zu Prozessionen, Waschungen, Lesungen und auch zur privaten Andacht und Meditation. Daher waren auch die Kapitelle mit ihrem bildlichen Schmuck und ihren Inschriften im täglichen Blickfeld der Kanoniker.

Der Zugang zur Kirche im Westen des Kreuzgangnordflügels wird wohl den Kanonikern gedient haben. Am Ostflügel lag wohl wie sonst auch üblich das Dormitorium, da wir wissen, dass es in späterer Zeit umgebaut wurde und wieder als Dormitorium diente. Im Erdgeschoss kann man den Kapitelsaal und das Auditorium vermuten. Der Zugang im Südflügel könnte der Zugang zum Refektorium gewesen sein.

Durch die Consuetudines von Rolduc wissen wir, dass es im Kreuzgang einen Ort gab, an dem die Novizen unterrichtet wurden. Die Unterweisung fand sicherlich nicht nur mit Büchern und durch Erzählungen statt, sondern sicherlich wurden auch die

$118 \mathrm{Zu}$ den Schwierigkeiten von Raumzuordnungen bei Regularkanonikerstiften siehe auch: Untermann 2016, 96-98.

119 Orlandoni 2001, 113-115.

120 Orlandoni 2001, 115 (Name); Zur Datierung siehe die Pläne 33 und 34 bei Bonnet/Perinetti 2001 zwischen den Seiten 32 und 33.

121 Orlandoni 2001, 113-115.

122 Auch vom Stift auf dem Petersberg bei Halle wissen wir von der Existenz von zwei Türen, die den Kreuzgang mit der Kirche verbanden. Hierdurch konnten auch die Laienbrüder den Kreuzgang problemlos aufsuchen. Siehe: Untermann 2016, 104. 
Kapitelle mit ihren figürlichen Szenen und Inschriften des gesamten Kreuzgangs eingebunden. An diesen lassen sich die Geschichten zeigen und erklären.

Die belehrende Funktion von Bildwerken haben bereits viele mittelalterliche Autoren wie Theophilus Presbyter, Honorius Augustodunensis oder Sicardus von Cremona beschrieben. ${ }^{123}$ Dass Bilder nicht nur der Belehrung dienten, wird durch den Kirchenlehrer Sicardus von Cremona deutlich, der in der Erinnerungsfunktion den eigentlichen Zweck der Bilder gesehen hat. ${ }^{124}$ So erinnerten die Kapitelle mit ihren Bildwerken sicherlich nicht nur Novizen und Kanoniker bei ihren täglichen Gängen und Aufenthalten im Kreuzgang, sondern auch Besucher an die entsprechenden Bibelstellen und die Geschichte des Konvents. Besonders eindrücklich dürfte es gewesen sein, wenn einem Novizen das erste Mal die Geschichte des heiligen Ursus beim Legendenkapitell erklärt wurde und plötzlich Wasser aus dem Loch des Brunnenreliefs floss. Hierzu war ein kleiner Kanal gebohrt worden und oben am Kapitell konnte man auf der anderen Seite Wasser in ein Loch eingießen. ${ }^{125}$

Auch die Inschriften werden die Novizen und Kanoniker an die entsprechenden Textpassagen bei den Propheten erinnert haben. Anhand der Prophetenkapitelle ist zu vermuten, dass die Mnemotechnik, die aus der Antike bekannt war, verwendet und gelehrt wurde. ${ }^{126}$ Eine Inschrift mit einem Auszug eines Bibelverses ruft die gesamte Bibelstelle in Erinnerung und die folgenden Inschriften genauso, sodass der gesamte Ablauf einer Geschichte in Erinnerung gebracht wird. Entscheidend ist hierbei die räumliche Anordnung der Bibelverse. Die Reihenfolge der Propheten auf den einzelnen Kapitellen entspricht der Reihenfolge in der Bibel. Bei der Mnemotechnik wird als Erinnerungshilfe ein imaginäres Gebäude mit Orten (loci) und Bildern verwendet. ${ }^{127}$ „Ein locus ist ein vom Gedächtnis leicht zu erfassender Ort, wie etwa ein Haus, ein Zwischenraum zwischen Säulen, eine Ecke, ein Bogen oder etwas Ähnliches. Bilder sind Formen, Zeichen oder Abbilder (formae, notae, simulacra) dessen, was erinnert werden soll." ${ }^{128}$ Die Säulen mit ihren Kapitellen in Aosta kann man sich hiernach als loci vorstellen und die Bilder bzw. Inschriften entsprechen den inneren Bildern im Gedächtnis und dienen somit als Einstieg zur Erinnerung der einzelnen Geschichten. Bei den Prophetenkapitellen in Aosta könnten zusätzlich die ausgewählten Versteile Hinweise auf eine Merkkette sein, bei der eventuell die ersten Buchstaben der einzel-

123 Theopilus Presbyter 2013, 246 (De diversis artibus, Prol. 3); Honorius Augustodunensis 1854, col. 586 (Gemma animae I, CXXXII); Sicard von Cremona 2008, 50 (Mitralis de officiis, lib. 1, cap. 12) 124 Siehe hierzu: Boerner 2008, 39.

125 Dass solche Spielereien zumindest im späteren Mittelalter durchaus üblich waren, konnte Johannes Tripps nachweisen. Siehe: Tripps 2000.

126 Zur Mnemonik im Mittelalter siehe: Yates 2012, 54-101; Boerner 2008, 49-52.

127 Yates 2012, 14-20.

128 Yates 2012, 15. 
nen Wörter wiederum andere Texte ins Gedächtnis rufen. Vielleicht mussten deswegen die besonders ausgewählten Versteile teilweise sogar verändert werden.

Somit ist es also durchaus wahrscheinlich, dass beim Kreuzgang von SS. Pietro ed Orso in Aosta Schrift und Bild der Bildung dienten.

\section{Literaturverzeichnis:}

Balthasar, Hans Urs von (1980), Die großen Ordensregeln (Lectio Spiritualis 12), 4. Auflage Einsiedeln.

Barberi, Sandra (1988), Il chiostro di S. Orso ad Aosta (Quaderni della Soprintendenza per i Beni Culturali della Valle d'Aosta, Nuova Serie 5), Rom.

Barberi, Sandra (2001), „Il chiostro“, in: Bruno Orlandoni u. Elena Rossetti Brezzi (Hgg.), Sant’Orso di Aosta. Il complesso monumentale, Aosta, Bd.1, 49-66.

Berton, Robert (1956), I capitelli del chiostro di Sant'Orso. Un gioiello d'arte romanica in Val d'Aosta, Novara.

Boerner, Bruno (2008), Bildwirkungen. Die kommunikative Funktion mittelalterlicher Skulpturen (Habil. Dresden 2003), Berlin.

Bonnet, Charles / Perinetti, Renato (2001), „La collegiata di Sant‘Orso dalle origine al XIII secolo“, in: Bruno Orlandoni u. Elena Rossetti Brezzi (Hgg.), Sant’Orso di Aosta. Il complesso monumentale, Aosta, Bd. 1, 9-34.

Debiais, Vincent (2009), Messages de pierre. La lecture des inscriptions dans la communication

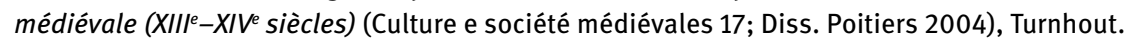

Dercks, Ute (2012), „Medievo interattivo: CENOBIUM - un progetto per i capitelli romanici nel Mediterrano“, in: Rosa Aloy (Hg.) Contextos 1200 i 1400. Art de Catalunya i art de l'Europa meridional en dos canvis de segle (Emac-contextos 2), Barcelona, 383-391.

Dickmann, Jens-Arne/Keil, Wilfried E. / Witschel, Christian (2015), „Topologie“, in: Thomas Meier, Michael R. Ott u. Rebecca Sauer (Hgg.), Materiale Textkulturen. Konzepte - Materialien - Praktiken (Materiale Textkulturen 1), Berlin / München / Boston, 113-128.

Donkin, Lucy (2008), „Ornata Decenter: Perceptions of ,fitting decoration“ amongst Augustinian canons of Sant'Orso in Aosta in the mid-twelfth century", in: Journal of the Warburg and Courtauld Institutes 71, 75-93.

„Expositio“ (1854), „Expositio in regulam beati Augustini“, in: Patrologiae cursus completus. Series latina, hg. v. Jacques Paul Migne, Bd. 176, Paris, 881-924.

Feraudi-Gruénais, Francisca (2017), „Das synaktive Potential von Beischriften“, in: Irene Berti, Katharina Bolle, Fanny Opdenhoff u. Fabian Stroth (Hgg.), Writing Matters. Presenting and Perceiving Monumental Inscriptions in Antiquity and the Middle Ages (Materiale Textkulturen 14), Berlin/ Boston 2017, 43-76.

Ferrari, Stella (2015), „St. Abbondio and St. Orso. Expressions of Devotion in Northern Italy through Architecture, Liturgical Space and Iconography“, in: Michele C. Ferrari (Hg.), Saints and the City. Beiträge zum Verständnis urbaner Sakralität in christlichen Gemeinschaften (5.-17. Jh.) (FAU Studien aus der Philosophischen Fakultät 3), Erlangen, 115-148.

Frese, Tobias (2006), Die Bildkritik des Bernhard von Clairvaux. Die Apologia im monastischen Diskurs, Bamberg.

Frese, Tobias/Keil, Wilfried E. / Krüger, Kristina (Hgg.) (2014), Verborgen, unsichtbar, unlesbar - zur Problematik restringierter Schriftpräsenz (Materiale Textkulturen 2), Berlin/Boston. 
Gandolfo, Francesco (1999), „Noterella in margine al chiostro die SS. Pietro e Orso ad Aosta“, in: Antonio Cadei (Hg.), Arte d'Occidente. Temi e Methodi. Studi in honore di Angiola Maria Romanini, Rom, Bd. 3, 369-372.

Heim, Manfred (2003a), „Augustinerchorherren“, in: Georg Schwaiger (Hg.), Mönchtum. Orden. Klöster. Von den Anfängen bis zur Gegenwart, München, 59-66.

Heim, Manfred (2003b), „Chorherren (Kanoniker)“, in: Georg Schwaiger (Hg.), Mönchtum. Orden. Klöster. Von den Anfängen bis zur Gegenwart, München, 131-146.

Hofmann, Heinz (1978), „Satorquadrat“, in: Paulys Realencyclopädie der classischen Altertumswissenschaft, Supplementband 15, München, 478-565.

Honorius Augustodunensis (1854), „Gemma animae“, in: Patrologiae cursus completus. Series latina, hg. v. Jacques Paul Migne, Bd.172, Paris, 541-738.

Jäggi, Carola (2007), „Die Kirche als heiliger Raum: Zur Geschichte eines Paradoxons“, in: Berndt Hamm, Klaus Herbers u. Heidrun Stein-Kecks (Hgg.), Sakralität zwischen Antike und Neuzeit (Beiträge zur Hagiographie 6), Stuttgart, 75-89.

Jullian, René (1964), „Une oeuvre lyonnaise de l'atelier du cloître d'Aoste“, in: Arte Lombarda 9, 1, 77-82.

Keil, Wilfried E. (2014), „Überlegungen zur restringierten Präsenz mittelalterlicher Bauinschriften“, in: Tobias Frese, Wilfried E. Keil u. Krüger, Kristina (Hgg.), Verborgen, unsichtbar, unlesbar - zur Problematik restringierter Schriftpräsenz (Materiale Textkulturen 2), Berlin / Boston, 117-142.

Keil, Wilfried E. (2018), „Remarks on patron inscriptions with restricted presence“, in: Jordi Camps, Manuel Castiñeiras, John Mc Neill u. Richard Plant (Hgg.), Romanesque Patrons and Processes. Design and Instrumentality in the Art and Architecture of Romanesque Europe (The British Archaeological Association Conference Transactions), London/ New York, 279-289.

Keil, Wilfried E./Kiyanrad, Sarah/Theiss, Christoffer/Willer, Laura (eds.) (2019), Zeichentragende Artefakte im sakralen Raum. Zwischen Präsenz und UnSichtbarkeit (Materiale Textkulturen 20), Berlin/Boston.

Krause, Hans-Joachim (1967), „Die romanische Klausur auf dem Petersberg bei Halle“, in: Elisabeth Hütter, Fritz Löffler u. Heinrich Magirius (Hgg.), Die Kunst des Mittelalters in Sachsen (Festschrift Wolf Schubert dargebracht zum sechzigsten Geburtstag am 28. Januar 1963), Weimar, 60-85.

Orlandoni, Bruno (2001), „Il complesso di Sant’Orso dal XIII al XV secolo“, in: Bruno Orlandoni u. Elena Rossetti Brezzi (Hgg.), Sant’Orso di Aosta. Il complesso monumentale, Aosta, Bd.1, 111-130.

Papone, Paolo/Vallet, Viviana (2001), „Il mosaico del coro“, in: Bruno Orlandoni u. Elena Rossetti Brezzi (Hgg.), Sant'Orso di Aosta. Il complesso monumentale, Aosta, Bd.1, 35-48.

Perinetti, Renato (2005), „Il mosaico del coro della chiesa dei Santi Pietro e Orso ad Aosta“, in: Hélène Morlier (Hg.), La mosaïque gréco-romaine (Collection de l'École Française de Rome 352; Colloque International pour l'Étude de la Mosaïque Antique et Médiévale, 9, 2001, Rom), Bd.1, 329-338.

Poeschke, Joachim (1998), Die Skulptur des Mittelalters in Italien, Bd. 1: Romanik, München.

Porter, Arthur Kingsely (1915-1917), Lombard Architecture, 4 Bde., New Haven/London / Oxford.

Pressouyre, Léon (1973), „St. Bernard to St. Francis. Monastic ideals and iconographic programs in the cloister", in: Gesta 12, 71-92.

Segre Montel, Costanza (2001), „La pittura romanica“, in: Bruno Orlandoni u. Elena Rossetti Brezzi (Hgg.), Sant'Orso di Aosta. Il complesso monumentale, Aosta, Bd.1, 79-100.

Sicard von Cremona (2008), Mitralis de officiis, hg. v. Gábor Sarbak u. Lorenz Weinrich (Corpus Christianorum. Continuatio Mediaevalis 228), Turnhout.

Theopilus Presbyter (2013), De diversis artibus, hg. v. Brepohl, Erhard: Theophilus Presbyter und das mittelalterliche Kunsthandwerk. Gesamtausgabe der Schrift De diversis artibus, 2. Aufl. Köln/ Weimar/Wien. 
Tripps, Johannes (2000), Das handelnde Bildwerk in der Gotik. Forschungen zu den Bedeutungsschichten und der Funktion des Kirchengebäudes und seiner Ausstattung in der Hoch- und Spätgotik (Habil. Heidelberg 1996), 2. Aufl. Berlin.

Untermann, Matthias (2009), Handbuch der mittelalterlichen Architektur, Darmstadt.

Untermann, Matthias (2016), „Häuser apostolischer Gemeinschaften. Die Konvente der Regularkanoniker“, in: Jörg Sonntag (Hg.), Geist und Gestalt. Monastische Raumkonzepte als Ausdrucksformen religiöser Leitideen im Mittelalter (Vita Regularis. Ordnungen und Deutungen religiösen Lebens im Mittelalter 69), 91-120.

Yates, Frances A. (2012), Gedächtnis und Erinnern. Mnemonik von Aristoteles bis Shakespeare, Berlin. (Englische Originalausgabe: The Art of Memory, London 1966).

\section{Bildnachweise}

Abb.1-15: Wilfried E. Keil. 
